\title{
Weighted estimates for vector-valued intrinsic square functions and commutators in the Morrey type spaces
}

\author{
Hua Wang * \\ College of Mathematics and Econometrics, Hunan University, Changsha 410082, P. R. China
}

\begin{abstract}
In this paper, the boundedness properties of vector-valued intrinsic square functions and their vector-valued commutators with $B M O\left(\mathbb{R}^{n}\right)$ functions are discussed. We first show the weighted strong type and weak type estimates of vector-valued intrinsic square functions in the Morrey type spaces. Then we obtain weighted strong type estimates of vectorvalued analogues of commutators in Morrey type spaces. In the endpoint case, we establish the weighted weak $L \log L$-type estimates for these vector-valued commutators in the setting of weighted Lebesgue spaces. Furthermore, we prove weighted endpoint estimates of these commutator operators in Morrey type spaces. In particular, we can obtain strong type and endpoint estimates of vector-valued intrinsic square functions and their commutators in the weighted Morrey spaces and the generalized Morrey spaces. MSC(2010): 42B25; 42B35

Keywords: Vector-valued intrinsic square functions; Morrey type spaces; vector-valued commutators; $A_{p}$ weights
\end{abstract}

\section{Introduction}

The intrinsic square functions were first introduced by Wilson in [18 19]; they are defined as follows. For $0<\alpha \leq 1$, let $\mathcal{C}_{\alpha}$ be the family of functions $\varphi: \mathbb{R}^{n} \longmapsto \mathbb{R}$ such that $\varphi$ 's support is contained in $\left\{x \in \mathbb{R}^{n}:|x| \leq 1\right\}, \int_{\mathbb{R}^{n}} \varphi(x) d x=0$, and for all $x, x^{\prime} \in \mathbb{R}^{n}$,

$$
\left|\varphi(x)-\varphi\left(x^{\prime}\right)\right| \leq\left|x-x^{\prime}\right|^{\alpha}
$$

For $(y, t) \in \mathbb{R}_{+}^{n+1}=\mathbb{R}^{n} \times(0,+\infty)$ and $f \in L_{l o c}^{1}\left(\mathbb{R}^{n}\right)$, we set

$$
A_{\alpha}(f)(y, t)=\sup _{\varphi \in \mathcal{C}_{\alpha}}\left|\varphi_{t} * f(y)\right|=\sup _{\varphi \in \mathcal{C}_{\alpha}}\left|\int_{\mathbb{R}^{n}} \varphi_{t}(y-z) f(z) d z\right|,
$$

*E-mail address: wanghua@pku.edu.cn. 
where $\varphi_{t}$ denotes the usual $L^{1}$ dilation of $\varphi: \varphi_{t}(y)=t^{-n} \varphi(y / t)$. Then we define the intrinsic square function of $f$ (of order $\alpha$ ) by the formula

$$
\mathcal{S}_{\alpha}(f)(x):=\left(\iint_{\Gamma(x)}\left(A_{\alpha}(f)(y, t)\right)^{2} \frac{d y d t}{t^{n+1}}\right)^{1 / 2},
$$

where $\Gamma(x)$ denotes the usual cone of aperture one:

$$
\Gamma(x):=\left\{(y, t) \in \mathbb{R}_{+}^{n+1}:|x-y|<t\right\} .
$$

This new function is independent of any particular kernel, and it dominates pointwise the classical square function (Lusin area integral) and its real-variable generalizations, one can see more details in [18,19]. Let $\vec{f}=\left(f_{1}, f_{2}, \ldots\right)$ be a sequence of locally integrable functions on $\mathbb{R}^{n}$. For any $x \in \mathbb{R}^{n}$, Wilson [19] also defined the vector-valued intrinsic square functions of $\vec{f}$ by

$$
\mathcal{S}_{\alpha}(\vec{f})(x):=\left(\sum_{j=1}^{\infty}\left|\mathcal{S}_{\alpha}\left(f_{j}\right)(x)\right|^{2}\right)^{1 / 2}
$$

In [19], Wilson has established the following two results.

Theorem A ([19]). Let $0<\alpha \leq 1,1<p<\infty$ and $w \in A_{p}$ (Muckenhoupt weight class). Then there exists a constant $C>0$ independent of $\vec{f}=\left(f_{1}, f_{2}, \ldots\right)$ such that

$$
\left\|\left(\sum_{j}\left|\mathcal{S}_{\alpha}\left(f_{j}\right)\right|^{2}\right)^{1 / 2}\right\|_{L_{w}^{p}} \leq C\left\|\left(\sum_{j}\left|f_{j}\right|^{2}\right)^{1 / 2}\right\|_{L_{w}^{p}} .
$$

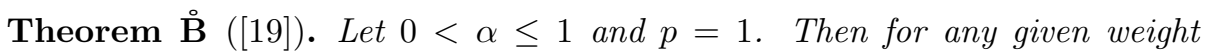
function $w$ and $\lambda>0$, there exists a constant $C>0$ independent of $\vec{f}=$ $\left(f_{1}, f_{2}, \ldots\right)$ and $\lambda>0$ such that

$w\left(\left\{x \in \mathbb{R}^{n}:\left(\sum_{j}\left|\mathcal{S}_{\alpha}\left(f_{j}\right)(x)\right|^{2}\right)^{1 / 2}>\lambda\right\}\right) \leq \frac{C}{\lambda} \int_{\mathbb{R}^{n}}\left(\sum_{j}\left|f_{j}(x)\right|^{2}\right)^{1 / 2} M(w)(x) d x$,

where $M$ denotes the standard Hardy-Littlewood maximal operator.

If we take $w \in A_{1}$, then $M(w)(x) \leq C \cdot w(x)$ for a.e. $x \in \mathbb{R}^{n}$ by the definition of $A_{1}$ weight (see Section 2). Hence, as a straightforward consequence of Theorem $\mathrm{B}$, we obtain

Theorem B. Let $0<\alpha \leq 1, p=1$ and $w \in A_{1}$. Then there exists a constant $C>0$ independent of $\vec{f}=\left(f_{1}, f_{2}, \ldots\right)$ such that

$$
\left\|\left(\sum_{j}\left|\mathcal{S}_{\alpha}\left(f_{j}\right)\right|^{2}\right)^{1 / 2}\right\|_{W L_{w}^{1}} \leq C\left\|\left(\sum_{j}\left|f_{j}\right|^{2}\right)^{1 / 2}\right\|_{L_{w}^{1}} .
$$


Let $b$ be a locally integrable function on $\mathbb{R}^{n}$ and $0<\alpha \leq 1$, the commutators generated by $b$ and intrinsic square functions $\mathcal{S}_{\alpha}$ are defined by the following expression in [16].

$$
\left[b, \mathcal{S}_{\alpha}\right](f)(x):=\left(\iint_{\Gamma(x)} \sup _{\varphi \in \mathcal{C}_{\alpha}}\left|\int_{\mathbb{R}^{n}}[b(x)-b(z)] \varphi_{t}(y-z) f(z) d z\right|^{2} \frac{d y d t}{t^{n+1}}\right)^{1 / 2} .
$$

In this paper, we will consider the vector-valued analogues of these commutator operators. Let $\vec{f}=\left(f_{1}, f_{2}, \ldots\right)$ be a sequence of locally integrable functions on $\mathbb{R}^{n}$. For any $x \in \mathbb{R}^{n}$, in the same way, we can define the commutators for vector-valued intrinsic square functions of $\vec{f}$ as

$$
\left[b, \mathcal{S}_{\alpha}\right](\vec{f})(x):=\left(\sum_{j=1}^{\infty}\left|\left[b, \mathcal{S}_{\alpha}\right]\left(f_{j}\right)(x)\right|^{2}\right)^{1 / 2} .
$$

On the other hand, the classical Morrey space was originally introduced by Morrey in 8 to study the local behavior of solutions to second order elliptic partial differential equations. Since then, this space played an important role in studying the regularity of solutions to partial differential equations. In [7, Mizuhara introduced the generalized Morrey space $L^{p, \Theta}\left(\mathbb{R}^{n}\right)$ which was later extended and studied by many authors. In [6], Komori and Shirai defined a version of the weighted Morrey space $L^{p, \kappa}(w)$ which is a natural generalization of weighted Lebesgue space. Recently, in [17, we have established the strong type and weak type estimates for vector-valued intrinsic square functions on $L^{p, \Theta}\left(\mathbb{R}^{n}\right)$ and $L^{p, \kappa}(w)$.

The main purpose of this paper is twofold. We first define a new kind of Morrey type spaces $\mathcal{M}^{p, \theta}(w)$ containing generalized Morrey space $L^{p, \Theta}\left(\mathbb{R}^{n}\right)$ and weighted Morrey space $L^{p, \kappa}(w)$ as special cases, and then we will discuss the boundedness properties of vector-valued intrinsic square functions (1.2) and vector-valued commutators (1.4) defined above in these Morrey type spaces $\mathcal{M}^{p, \theta}(w)$ for all $1 \leq p<\infty$.

\section{Main results}

\subsection{Notations and preliminaries}

A weight $w$ will always mean a positive function which is locally integrable on $\mathbb{R}^{n}, B=B\left(x_{0}, r_{B}\right)=\left\{x \in \mathbb{R}^{n}:\left|x-x_{0}\right|<r_{B}\right\}$ denotes the open ball centered at $x_{0}$ and with radius $r_{B}>0$. For $1<p<\infty$, a weight function $w$ is said to belong to the Muckenhoupt's class $A_{p}$, if there is a constant $C>0$ such that for every ball $B \subseteq \mathbb{R}^{n}$ (see [4,9]),

$$
\left(\frac{1}{|B|} \int_{B} w(x) d x\right)^{1 / p}\left(\frac{1}{|B|} \int_{B} w(x)^{-p^{\prime} / p} d x\right)^{1 / p^{\prime}} \leq C,
$$


where $p^{\prime}$ is the dual of $p$ such that $1 / p+1 / p^{\prime}=1$. For the case $p=1, w \in A_{1}$, if there is a constant $C>0$ such that for every ball $B \subseteq \mathbb{R}^{n}$,

$$
\frac{1}{|B|} \int_{B} w(x) d x \leq C \cdot \underset{x \in B}{\operatorname{essinf}} w(x) .
$$

We also define $A_{\infty}=\bigcup_{1<p<\infty} A_{p}$. It is well known that if $w \in A_{p}$ with $1 \leq p<\infty$, then for any ball $B$, there exists an absolute constant $C>0$ such that

$$
w(2 B) \leq C \cdot w(B) .
$$

In general, for $w \in A_{1}$ and any $j \in \mathbb{Z}_{+}$, there exists an absolute constant $C>0$ such that (see 4])

$$
w\left(2^{j} B\right) \leq C \cdot 2^{j n} w(B) .
$$

Moreover, if $w \in A_{\infty}$, then for all balls $B$ and all measurable subsets $E$ of $B$, there exists a number $\delta>0$ independent of $E$ and $B$ such that (see [4])

$$
\frac{w(E)}{w(B)} \leq C\left(\frac{|E|}{|B|}\right)^{\delta} .
$$

A weight function $w$ is said to belong to the reverse Hölder class $R H_{r}$, if there exist two constants $r>1$ and $C>0$ such that the following reverse Hölder inequality holds for every ball $B \subseteq \mathbb{R}^{n}$.

$$
\left(\frac{1}{|B|} \int_{B} w(x)^{r} d x\right)^{1 / r} \leq C\left(\frac{1}{|B|} \int_{B} w(x) d x\right) .
$$

Given a ball $B$ and $\lambda>0, \lambda B$ denotes the ball with the same center as $B$ whose radius is $\lambda$ times that of $B$. For a given weight function $w$ and a measurable set $E$, we also denote the Lebesgue measure of $E$ by $|E|$ and the weighted measure of $E$ by $w(E)$, where $w(E)=\int_{E} w(x) d x$. Equivalently, we could define the above notions with cubes instead of balls. Hence we shall use these two different definitions appropriate to calculations.

Given a weight function $w$ on $\mathbb{R}^{n}$, for $1 \leq p<\infty$, the weighted Lebesgue space $L_{w}^{p}\left(\mathbb{R}^{n}\right)$ is defined as the set of all functions $f$ such that

$$
\|f\|_{L_{w}^{p}}:=\left(\int_{\mathbb{R}^{n}}|f(x)|^{p} w(x) d x\right)^{1 / p}<\infty .
$$

We also denote by $W L_{w}^{1}\left(\mathbb{R}^{n}\right)$ the weighted weak Lebesgue space consisting of all measurable functions $f$ such that

$$
\|f\|_{W L_{w}^{1}}:=\sup _{\lambda>0} \lambda \cdot w\left(\left\{x \in \mathbb{R}^{n}:|f(x)|>\lambda\right\}\right)<\infty .
$$

We next recall some basic definitions and facts about Orlicz spaces needed for the proofs of the main results. For more information on the subject, one can see [14]. A function $\Phi$ is called a Young function if it is continuous, nonnegative, 
convex and strictly increasing on $[0,+\infty)$ with $\Phi(0)=0$ and $\Phi(t) \rightarrow+\infty$ as $t \rightarrow+\infty$. We define the $\Phi$-average of a function $f$ over a ball $B$ by means of the following Luxemburg norm:

$$
\|f\|_{\Phi, B}:=\inf \left\{\sigma>0: \frac{1}{|B|} \int_{B} \Phi\left(\frac{|f(x)|}{\sigma}\right) d x \leq 1\right\} .
$$

An equivalent norm that is often useful in calculations is as follows(see [10,14]):

$$
\|f\|_{\Phi, B} \leq \inf _{\eta>0}\left\{\eta+\frac{\eta}{|B|} \int_{B} \Phi\left(\frac{|f(x)|}{\eta}\right) d x\right\} \leq 2\|f\|_{\Phi, B} .
$$

Given a Young function $\Phi$, we use $\bar{\Phi}$ to denote the complementary Young function associated to $\Phi$. Then the following generalized Hölder's inequality holds for any given ball $B$ (see [10,11]).

$$
\frac{1}{|B|} \int_{B}|f(x) \cdot g(x)| d x \leq 2\|f\|_{\Phi, B}\|g\|_{\bar{\Phi}, B} .
$$

In order to deal with the weighted case, for $w \in A_{\infty}$, we need to define the weighted $\Phi$-average of a function $f$ over a ball $B$ by means of the weighted Luxemburg norm:

$$
\|f\|_{\Phi(w), B}:=\inf \left\{\sigma>0: \frac{1}{w(B)} \int_{B} \Phi\left(\frac{|f(x)|}{\sigma}\right) w(x) d x \leq 1\right\} .
$$

It can be shown that for $w \in A_{\infty}$ (see [14,20]),

$$
\|f\|_{\Phi(w), B} \approx \inf _{\eta>0}\left\{\eta+\frac{\eta}{w(B)} \int_{B} \Phi\left(\frac{|f(x)|}{\eta}\right) w(x) d x\right\},
$$

and

$$
\frac{1}{w(B)} \int_{B}|f(x) \cdot g(x)| w(x) d x \leq C\|f\|_{\Phi(w), B}\|g\|_{\bar{\Phi}(w), B} .
$$

The young function that we are going to use is $\Phi(t)=t \cdot\left(1+\log ^{+} t\right)$ with its complementary Young function $\bar{\Phi}(t) \approx \exp (t)$. Here by $A \approx B$, we mean that there exists a constant $C>1$ such that $\frac{1}{C} \leq \frac{A}{B} \leq C$. In the present situation, we denote

$$
\|f\|_{L \log L(w), B}=\|f\|_{\Phi(w), B}, \quad\|g\|_{\exp L(w), B}=\|g\|_{\bar{\Phi}(w), B} .
$$

By the generalized Hölder's inequality with weight, we have (see [10, 20])

$$
\frac{1}{w(B)} \int_{B}|f(x) \cdot g(x)| w(x) d x \leq C\|f\|_{L \log L(w), B}\|g\|_{\exp L(w), B} .
$$

Let us now recall the definition of the space of $B M O\left(\mathbb{R}^{n}\right)$ (Bounded Mean Oscillation) (see 35 ). A locally integrable function $b$ is said to be in $B M O\left(\mathbb{R}^{n}\right)$, if

$$
\|b\|_{*}:=\sup _{B} \frac{1}{|B|} \int_{B}\left|b(x)-b_{B}\right| d x<\infty
$$


where $b_{B}$ stands for the average of $b$ on $B$, i.e., $b_{B}=\frac{1}{|B|} \int_{B} b(y) d y$ and the supremum is taken over all balls $B$ in $\mathbb{R}^{n}$. Modulo constants, the space $B M O\left(\mathbb{R}^{n}\right)$ is a Banach space with respect to the norm $\|\cdot\|_{*}$. By the John-Nirenberg's inequality, it is not difficult to see that for any $w \in A_{\infty}$ and any given ball $B$ (see [20]),

$$
\left\|b-b_{B}\right\|_{\exp L(w), B} \leq C\|b\|_{*} .
$$

\section{$2.2 \quad$ Morrey type spaces}

Definition 2.1 (白). Let $1 \leq p<\infty, 0<\kappa<1$ and $w$ be a weight function on $\mathbb{R}^{n}$. Then the weighted Morrey space $L^{p, \kappa}(w)$ is defined by

$L^{p, \kappa}(w):=\left\{f \in L_{l o c}^{p}(w):\|f\|_{L^{p, \kappa}(w)}=\sup _{B}\left(\frac{1}{w(B)^{\kappa}} \int_{B}|f(x)|^{p} w(x) d x\right)^{1 / p}<\infty\right\}$,

where the supremum is taken over all balls $B$ in $\mathbb{R}^{n}$. We also denote by $W L^{1, \kappa}(w)$ the weighted weak Morrey space of all measurable functions $f$ such that

$$
\sup _{B} \sup _{\lambda>0} \frac{1}{w(B)^{\kappa}} \lambda \cdot w(\{x \in B:|f(x)|>\lambda\}) \leq C<\infty .
$$

Let $\Theta=\Theta(r), r>0$, be a growth function, that is, a positive increasing function in $(0,+\infty)$ and satisfy the following doubling condition:

$$
\Theta(2 r) \leq D \cdot \Theta(r), \quad \text { for all } r>0,
$$

where $D=D(\Theta) \geq 1$ is a doubling constant independent of $r$.

Definition 2.2 (7]). Let $1 \leq p<\infty$ and $\Theta$ be a growth function in $(0,+\infty)$. Then the generalized Morrey space $L^{p, \Theta}\left(\mathbb{R}^{n}\right)$ is defined as the set of all locally integrable functions $f$ for which

$$
\|f\|_{L^{p, \Theta}}:=\sup _{r>0 ; B\left(x_{0}, r\right)}\left(\frac{1}{\Theta(r)} \int_{B\left(x_{0}, r\right)}|f(x)|^{p} d x\right)^{1 / p}<\infty,
$$

where the supremum is taken over all balls $B\left(x_{0}, r\right)$ in $\mathbb{R}^{n}$ with $x_{0} \in \mathbb{R}^{n}$. We also denote by $W L^{1, \Theta}\left(\mathbb{R}^{n}\right)$ the generalized weak Morrey space of all measurable functions $f$ for which

$$
\sup _{B\left(x_{0}, r\right)} \sup _{\lambda>0} \frac{1}{\Theta(r)} \lambda \cdot\left|\left\{x \in B\left(x_{0}, r\right):|f(x)|>\lambda\right\}\right| \leq C<\infty .
$$

In order to unify these two definitions, we will introduce Morrey type spaces as follows. Let $0 \leq \kappa<1$. Assume that $\theta(\cdot)$ is a positive increasing function defined in $(0,+\infty)$ and satisfies the following $\mathcal{D}_{\kappa}$ condition:

$$
\frac{\theta(\xi)}{\xi^{\kappa}} \leq C \cdot \frac{\theta\left(\xi^{\prime}\right)}{\left(\xi^{\prime}\right)^{\kappa}}, \quad \text { for any } 0<\xi^{\prime}<\xi<+\infty,
$$

where $C>0$ is a constant independent of $\xi$ and $\xi^{\prime}$. 
Definition 2.3. Let $1 \leq p<\infty, 0 \leq \kappa<1, \theta$ satisfy the $\mathcal{D}_{\kappa}$ condition (2.9) and $w$ be a weight function on $\mathbb{R}^{n}$. We denote by $\mathcal{M}^{p, \theta}(w)$ the generalized weighted Morrey space of all locally integrable functions $f$ defined on $\mathbb{R}^{n}$, such that for every ball $B$ in $\mathbb{R}^{n}$,

$$
\left(\frac{1}{\theta(w(B))} \int_{B}|f(x)|^{p} w(x) d x\right)^{1 / p} \leq C<\infty .
$$

Then we let $\|f\|_{\mathcal{M}^{p, \theta}(w)}$ be the smallest constant $C>0$ satisfying the above estimate and $\mathcal{M}^{p, \theta}(w)$ becomes a Banach function space with norm $\|\cdot\|_{\mathcal{M}^{p, \theta}(w)}$. In the unweighted case(when $w$ equals a constant function), we denote the generalized unweighted Morrey space by $\mathcal{M}^{p, \theta}\left(\mathbb{R}^{n}\right)$. That is, let $1 \leq p<\infty$ and $\theta$ satisfy the $\mathcal{D}_{\kappa}$ condition (2.9) with $0 \leq \kappa<1$, we define

$\mathcal{M}^{p, \theta}\left(\mathbb{R}^{n}\right):=\left\{f \in L_{l o c}^{p}\left(\mathbb{R}^{n}\right):\|f\|_{\mathcal{M}^{p, \theta}}=\sup _{B}\left(\frac{1}{\theta(|B|)} \int_{B}|f(x)|^{p} d x\right)^{1 / p}<\infty\right\}$.

Definition 2.4. Let $p=1,0 \leq \kappa<1, \theta$ satisfy the $\mathcal{D}_{\kappa}$ condition (2.9) and $w$ be a weight function on $\mathbb{R}^{n}$. We denote by $W \mathcal{M}^{1, \theta}(w)$ the generalized weighted weak Morrey space consisting of all measurable functions $f$ defined on $\mathbb{R}^{n}$ for which

$$
\|f\|_{W \mathcal{M}^{1, \theta}(w)}:=\sup _{B} \sup _{\sigma>0} \frac{1}{\theta(w(B))} \sigma \cdot w(\{x \in B:|f(x)|>\sigma\}) \leq C<\infty .
$$

In the unweighted case(when $w$ equals a constant function), we denote the generalized unweighted weak Morrey space by $W \mathcal{M}^{1, \theta}\left(\mathbb{R}^{n}\right)$. That is, let $p=1$ and $\theta$ satisfy the $\mathcal{D}_{\kappa}$ condition (2.9) with $0 \leq \kappa<1$, we define

$W \mathcal{M}^{1, \theta}\left(\mathbb{R}^{n}\right):=\left\{f:\|f\|_{W \mathcal{M}^{1, \theta}}=\sup _{B} \sup _{\sigma>0} \frac{1}{\theta(|B|)} \sigma \cdot|\{x \in B:|f(x)|>\sigma\}|<\infty\right\}$.

Note that

- If $\theta(x) \equiv 1$, then $\mathcal{M}^{p, \theta}(w)=L_{w}^{p}\left(\mathbb{R}^{n}\right)$ and $W \mathcal{M}^{p, \theta}(w)=W L_{w}^{p}\left(\mathbb{R}^{n}\right)$. Thus our (weak) Morrey type space is an extension of the weighted (weak) Lebesgue space;

- If $\theta(x)=x^{\kappa}$ with $0<\kappa<1$, then $\mathcal{M}^{p, \theta}(w)$ is just the weighted Morrey space $L^{p, \kappa}(w)$, and $W \mathcal{M}^{1, \theta}(w)$ is just the weighted weak Morrey space $W L^{1, \kappa}(w)$;

- If $w$ equals a constant function, below we will show that $\mathcal{M}^{p, \theta}\left(\mathbb{R}^{n}\right)$ reduces to the generalized Morrey space $L^{p, \Theta}\left(\mathbb{R}^{n}\right)$, and $W \mathcal{M}^{1, \theta}\left(\mathbb{R}^{n}\right)$ reduces to the generalized weak Morrey space $W L^{1, \Theta}\left(\mathbb{R}^{n}\right)$. 


\subsection{Main theorems}

The main results of this paper can be stated as follows.

Theorem 2.1. Let $0<\alpha \leq 1,1<p<\infty$ and $w \in A_{p}$. Assume that $\theta$ satisfies the $\mathcal{D}_{\kappa}$ condition (2.9) with $0 \leq \kappa<1$, then there is a constant $C>0$ independent of $\vec{f}=\left(f_{1}, f_{2}, \ldots\right)$ such that

$$
\left\|\left(\sum_{j}\left|\mathcal{S}_{\alpha}\left(f_{j}\right)\right|^{2}\right)^{1 / 2}\right\|_{\mathcal{M}^{p, \theta}(w)} \leq C\left\|\left(\sum_{j}\left|f_{j}\right|^{2}\right)^{1 / 2}\right\|_{\mathcal{M}^{p, \theta}(w)} .
$$

Theorem 2.2. Let $0<\alpha \leq 1, p=1$ and $w \in A_{1}$. Assume that $\theta$ satisfies the $\mathcal{D}_{\kappa}$ condition (2.9) with $0 \leq \kappa<1$, then there is a constant $C>0$ independent of $\vec{f}=\left(f_{1}, f_{2}, \ldots\right)$ such that

$$
\left\|\left(\sum_{j}\left|\mathcal{S}_{\alpha}\left(f_{j}\right)\right|^{2}\right)^{1 / 2}\right\|_{W \mathcal{M}^{1, \theta}(w)} \leq C\left\|\left(\sum_{j}\left|f_{j}\right|^{2}\right)^{1 / 2}\right\|_{\mathcal{M}^{1, \theta}(w)} .
$$

Theorem 2.3. Let $0<\alpha \leq 1,1<p<\infty, w \in A_{p}$ and $b \in \operatorname{BMO}\left(\mathbb{R}^{n}\right)$. Assume that $\theta$ satisfies the $\mathcal{D}_{\kappa}$ condition (2.9) with $0 \leq \kappa<1$, then there is a constant $C>0$ independent of $\vec{f}=\left(f_{1}, f_{2}, \ldots\right)$ such that

$$
\left\|\left(\sum_{j}\left|\left[b, \mathcal{S}_{\alpha}\right]\left(f_{j}\right)\right|^{2}\right)^{1 / 2}\right\|_{\mathcal{M}^{p, \theta}(w)} \leq C\left\|\left(\sum_{j}\left|f_{j}\right|^{2}\right)^{1 / 2}\right\|_{\mathcal{M}^{p, \theta}(w)} .
$$

In order to simplify the notations, for any given $\sigma>0$, we set

$$
\Phi\left(\frac{|f(x)|}{\sigma}\right)=\frac{|f(x)|}{\sigma} \cdot\left(1+\log +\frac{|f(x)|}{\sigma}\right)
$$

when $\Phi(t)=t \cdot\left(1+\log ^{+} t\right)$ and $\log ^{+} t=\max \{\log t, 0\}$. For the endpoint estimates for these commutator operators in the weighted Lebesgue space $L_{w}^{1}\left(\mathbb{R}^{n}\right)$, we will show

Theorem 2.4. Let $0<\alpha \leq 1, p=1, w \in A_{1}$ and $b \in B M O\left(\mathbb{R}^{n}\right)$. Then for any given $\sigma>0$, there exists a constant $C>0$ independent of $\vec{f}=\left(f_{1}, f_{2}, \ldots\right)$ and $\sigma>0$ such that

$w\left(\left\{x \in \mathbb{R}^{n}:\left(\sum_{j}\left|\left[b, \mathcal{S}_{\alpha}\right]\left(f_{j}\right)(x)\right|^{2}\right)^{1 / 2}>\sigma\right\}\right) \leq C \int_{\mathbb{R}^{n}} \Phi\left(\frac{\|\vec{f}(x)\|_{\ell^{2}}}{\sigma}\right) \cdot w(x) d x$

where $\Phi(t)=t \cdot\left(1+\log ^{+} t\right)$ and $\|\vec{f}(x)\|_{\ell^{2}}=\left(\sum_{j}\left|f_{j}(x)\right|^{2}\right)^{1 / 2}$.

For the endpoint estimates of commutators generated by $B M O\left(\mathbb{R}^{n}\right)$ functions and vector-valued intrinsic square functions in the Morrey type spaces associated to $\theta$, we will prove 
Theorem 2.5. Let $0<\alpha \leq 1, p=1, w \in A_{1}$ and $b \in B M O\left(\mathbb{R}^{n}\right)$. Assume that $\theta$ satisfies the $\mathcal{D}_{\kappa}$ condition (2.9) with $0 \leq \kappa<1$, then for any given $\sigma>0$ and any ball $B$ in $\mathbb{R}^{n}$, there exists a constant $C>0$ independent of $\vec{f}=\left(f_{1}, f_{2}, \ldots\right)$, $B$ and $\sigma>0$ such that

$$
\begin{aligned}
& \frac{1}{\theta(w(B))} \cdot w\left(\left\{x \in B:\left(\sum_{j}\left|\left[b, \mathcal{S}_{\alpha}\right]\left(f_{j}\right)(x)\right|^{2}\right)^{1 / 2}>\sigma\right\}\right) \\
\leq & C \cdot \sup _{B}\left\{\frac{\Phi\left(\frac{w(B)}{\theta(w(B))}\right)}{w(B)} \int_{B} \Phi\left(\frac{\|\vec{f}(x)\|_{\ell^{2}}}{\sigma}\right) \cdot w(x) d x\right\},
\end{aligned}
$$

where $\Phi(t)=t \cdot\left(1+\log ^{+} t\right)$ and $\|\vec{f}(x)\|_{\ell^{2}}=\left(\sum_{j}\left|f_{j}(x)\right|^{2}\right)^{1 / 2}$.

In particular, if we take $\theta(x)=x^{\kappa}$ with $0<\kappa<1$, then we immediately get the following strong type estimates and endpoint estimates of vector-valued intrinsic square functions and commutators in the weighted Morrey spaces $L^{p, \kappa}(w)$ for all $0<\kappa<1$ and $1 \leq p<\infty$.

Corollary 2.6. Let $0<\alpha \leq 1,1<p<\infty, 0<\kappa<1$ and $w \in A_{p}$. Then there is a constant $C>0$ independent of $\vec{f}=\left(f_{1}, f_{2}, \ldots\right)$ such that

$$
\left\|\left(\sum_{j}\left|\mathcal{S}_{\alpha}\left(f_{j}\right)\right|^{2}\right)^{1 / 2}\right\|_{L^{p, \kappa}(w)} \leq C\left\|\left(\sum_{j}\left|f_{j}\right|^{2}\right)^{1 / 2}\right\|_{L^{p, \kappa}(w)} .
$$

Corollary 2.7. Let $0<\alpha \leq 1, p=1,0<\kappa<1$ and $w \in A_{1}$. Then there is a constant $C>0$ independent of $\vec{f}=\left(f_{1}, f_{2}, \ldots\right)$ such that

$$
\left\|\left(\sum_{j}\left|\mathcal{S}_{\alpha}\left(f_{j}\right)\right|^{2}\right)^{1 / 2}\right\|_{W L^{1, \kappa}(w)} \leq C\left\|\left(\sum_{j}\left|f_{j}\right|^{2}\right)^{1 / 2}\right\|_{L^{1, \kappa}(w)} .
$$

Corollary 2.8. Let $0<\alpha \leq 1,1<p<\infty, 0<\kappa<1, w \in A_{p}$ and $b \in B M O\left(\mathbb{R}^{n}\right)$. Then there is a constant $C>0$ independent of $\vec{f}=\left(f_{1}, f_{2}, \ldots\right)$ such that

$$
\left\|\left(\sum_{j}\left|\left[b, \mathcal{S}_{\alpha}\right]\left(f_{j}\right)\right|^{2}\right)^{1 / 2}\right\|_{L^{p, \kappa}(w)} \leq C\left\|\left(\sum_{j}\left|f_{j}\right|^{2}\right)^{1 / 2}\right\|_{L^{p, \kappa}(w)} .
$$

Corollary 2.9. Let $0<\alpha \leq 1, p=1,0<\kappa<1, w \in A_{1}$ and $b \in B M O\left(\mathbb{R}^{n}\right)$. Then for any given $\sigma>0$ and any ball $B$ in $\mathbb{R}^{n}$, there exists a constant $C>0$ independent of $\vec{f}=\left(f_{1}, f_{2}, \ldots\right), B$ and $\sigma>0$ such that

$$
\begin{aligned}
& \frac{1}{w(B)^{\kappa}} \cdot w\left(\left\{x \in B:\left(\sum_{j}\left|\left[b, \mathcal{S}_{\alpha}\right]\left(f_{j}\right)(x)\right|^{2}\right)^{1 / 2}>\sigma\right\}\right) \\
\leq & C \cdot \sup _{B}\left\{\frac{\Phi\left(w(B)^{1-\kappa}\right)}{w(B)} \int_{B} \Phi\left(\frac{\|\vec{f}(x)\|_{\ell^{2}}}{\sigma}\right) \cdot w(x) d x\right\},
\end{aligned}
$$


where $\Phi(t)=t \cdot\left(1+\log ^{+} t\right)$ and $\|\vec{f}(x)\|_{\ell^{2}}=\left(\sum_{j}\left|f_{j}(x)\right|^{2}\right)^{1 / 2}$.

We can also take $w$ to be a constant function, then we immediately get the following unweighted results.

Corollary 2.10. Let $0<\alpha \leq 1$ and $1<p<\infty$. Assume that $\theta$ satisfies the $\mathcal{D}_{\kappa}$ condition (2.9) with $0 \leq \kappa<1$, then there is a constant $C>0$ independent of $\vec{f}=\left(f_{1}, f_{2}, \ldots\right)$ such that

$$
\left\|\left(\sum_{j}\left|\mathcal{S}_{\alpha}\left(f_{j}\right)\right|^{2}\right)^{1 / 2}\right\|_{\mathcal{M}^{p, \theta}} \leq C\left\|\left(\sum_{j}\left|f_{j}\right|^{2}\right)^{1 / 2}\right\|_{\mathcal{M}^{p, \theta}} .
$$

Corollary 2.11. Let $0<\alpha \leq 1$ and $p=1$. Assume that $\theta$ satisfies the $\mathcal{D}_{\kappa}$ condition (2.9) with $0 \leq \kappa<1$, then there is a constant $C>0$ independent of $\vec{f}=\left(f_{1}, f_{2}, \ldots\right)$ such that

$$
\left\|\left(\sum_{j}\left|\mathcal{S}_{\alpha}\left(f_{j}\right)\right|^{2}\right)^{1 / 2}\right\|_{W \mathcal{M}^{1, \theta}} \leq C\left\|\left(\sum_{j}\left|f_{j}\right|^{2}\right)^{1 / 2}\right\|_{\mathcal{M}^{1, \theta}} .
$$

Corollary 2.12. Let $0<\alpha \leq 1,1<p<\infty$ and $b \in B M O\left(\mathbb{R}^{n}\right)$. Assume that $\theta$ satisfies the $\mathcal{D}_{\kappa}$ condition (2.9) with $0 \leq \kappa<1$, then there is a constant $C>0$ independent of $\vec{f}=\left(f_{1}, f_{2}, \ldots\right)$ such that

$$
\left\|\left(\sum_{j}\left|\left[b, \mathcal{S}_{\alpha}\right]\left(f_{j}\right)\right|^{2}\right)^{1 / 2}\right\|_{\mathcal{M}^{p, \theta}} \leq C\left\|\left(\sum_{j}\left|f_{j}\right|^{2}\right)^{1 / 2}\right\|_{\mathcal{M}^{p, \theta}} .
$$

Corollary 2.13. Let $0<\alpha \leq 1, p=1$ and $b \in B M O\left(\mathbb{R}^{n}\right)$. Assume that $\theta$ satisfies the $\mathcal{D}_{\kappa}$ condition (2.9) with $0 \leq \kappa<1$, then for any given $\sigma>0$ and any ball $B$ in $\mathbb{R}^{n}$, there exists a constant $C>0$ independent of $\vec{f}=\left(f_{1}, f_{2}, \ldots\right)$, $B$ and $\sigma>0$ such that

$$
\begin{aligned}
& \frac{1}{\theta(|B|)} \cdot\left|\left\{x \in B:\left(\sum_{j}\left|\left[b, \mathcal{S}_{\alpha}\right]\left(f_{j}\right)(x)\right|^{2}\right)^{1 / 2}>\sigma\right\}\right| \\
\leq & C \cdot \sup _{B}\left\{\frac{\Phi\left(\frac{|B|}{\theta(|B|)}\right)}{|B|} \int_{B} \Phi\left(\frac{\|\vec{f}(x)\|_{\ell^{2}}}{\sigma}\right) d x\right\},
\end{aligned}
$$

where $\Phi(t)=t \cdot\left(1+\log ^{+} t\right)$ and $\|\vec{f}(x)\|_{\ell^{2}}=\left(\sum_{j}\left|f_{j}(x)\right|^{2}\right)^{1 / 2}$.

Let $\Theta=\Theta(r), r>0$, be a growth function with doubling constant $D(\Theta)$ : $1 \leq D(\Theta)<2^{n}$. If for any fixed $x_{0} \in \mathbb{R}^{n}$, we set $\theta\left(\left|B\left(x_{0}, r\right)\right|\right)=\Theta(r)$, then

$$
\theta\left(2^{n}\left|B\left(x_{0}, r\right)\right|\right)=\theta\left(\left|B\left(x_{0}, 2 r\right)\right|\right)=\Theta(2 r) .
$$

For the doubling constant $D(\Theta)$ satisfying $1 \leq D(\Theta)<2^{n}$, which means that $D(\Theta)=2^{\kappa \cdot n}$ for some $0 \leq \kappa<1$, then we are able to verify that $\theta$ is an increasing 
function and satisfies the $\mathcal{D}_{\kappa}$ condition (2.9) with some $0 \leq \kappa<1$. Thus, by the above unweighted results(Corollaries 2.10 through 2.13), we can also obtain strong type estimates and endpoint estimates of vector-valued intrinsic square functions and commutators in the generalized Morrey spaces $L^{p, \Theta}\left(\mathbb{R}^{n}\right)$ when $1 \leq p<\infty$ and $\Theta$ satisfies the doubling condition (2.8).

Corollary 2.14. Let $0<\alpha \leq 1$ and $1<p<\infty$. Suppose that $\Theta$ satisfies the doubling condition (2.8) and $1 \leq D(\Theta)<2^{n}$, then there is a constant $C>0$ independent of $\vec{f}=\left(f_{1}, f_{2}, \ldots\right)$ such that

$$
\left\|\left(\sum_{j}\left|\mathcal{S}_{\alpha}\left(f_{j}\right)\right|^{2}\right)^{1 / 2}\right\|_{L^{p, \Theta}} \leq C\left\|\left(\sum_{j}\left|f_{j}\right|^{2}\right)^{1 / 2}\right\|_{L^{p, \Theta}} .
$$

Corollary 2.15. Let $0<\alpha \leq 1$ and $p=1$. Suppose that $\Theta$ satisfies the doubling condition (2.8) and $1 \leq D(\Theta)<2^{n}$, then there is a constant $C>0$ independent of $\vec{f}=\left(f_{1}, f_{2}, \ldots\right)$ such that

$$
\left\|\left(\sum_{j}\left|\mathcal{S}_{\alpha}\left(f_{j}\right)\right|^{2}\right)^{1 / 2}\right\|_{W L^{1, \Theta}} \leq C\left\|\left(\sum_{j}\left|f_{j}\right|^{2}\right)^{1 / 2}\right\|_{L^{1, \Theta}} .
$$

Corollary 2.16. Let $0<\alpha \leq 1,1<p<\infty$ and $b \in B M O\left(\mathbb{R}^{n}\right)$. Suppose that $\Theta$ satisfies the doubling condition (2.8) and $1 \leq D(\Theta)<2^{n}$, then there is a constant $C>0$ independent of $\vec{f}=\left(f_{1}, f_{2}, \ldots\right)$ such that

$$
\left\|\left(\sum_{j}\left|\left[b, \mathcal{S}_{\alpha}\right]\left(f_{j}\right)\right|^{2}\right)^{1 / 2}\right\|_{L^{p, \Theta}} \leq C\left\|\left(\sum_{j}\left|f_{j}\right|^{2}\right)^{1 / 2}\right\|_{L^{p, \Theta}} .
$$

Corollary 2.17. Let $0<\alpha \leq 1, p=1$ and $b \in B M O\left(\mathbb{R}^{n}\right)$. Suppose that $\Theta$ satisfies the doubling condition (2.8) and $1 \leq D(\Theta)<2^{n}$, then for any given $\sigma>0$ and any ball $B\left(x_{0}, r\right)$ in $\mathbb{R}^{n}$, there exists a constant $C>0$ independent of $\vec{f}=\left(f_{1}, f_{2}, \ldots\right), B\left(x_{0}, r\right)$ and $\sigma>0$ such that

$$
\begin{aligned}
& \frac{1}{\Theta(r)} \cdot\left|\left\{x \in B\left(x_{0}, r\right):\left(\sum_{j}\left|\left[b, \mathcal{S}_{\alpha}\right]\left(f_{j}\right)(x)\right|^{2}\right)^{1 / 2}>\sigma\right\}\right| \\
\leq & C \cdot \sup _{r>0}\left\{\frac{\Phi\left(\frac{\left|B\left(x_{0}, r\right)\right|}{\Theta(r)}\right)}{\left|B\left(x_{0}, r\right)\right|} \int_{B\left(x_{0}, r\right)} \Phi\left(\frac{\|\vec{f}(x)\|_{\ell^{2}}}{\sigma}\right) d x\right\},
\end{aligned}
$$

where $\Phi(t)=t \cdot\left(1+\log ^{+} t\right)$ and $\|\vec{f}(x)\|_{\ell^{2}}=\left(\sum_{j}\left|f_{j}(x)\right|^{2}\right)^{1 / 2}$.

Throughout this paper, the letter $C$ always denotes a positive constant independent of the main parameters involved, but it may be different from line to line. 


\section{Proofs of Theorems 2.1 and 2.2}

Proof of Theorem 2.1. Let $\left(\sum_{j}\left|f_{j}\right|^{2}\right)^{1 / 2} \in \mathcal{M}^{p, \theta}(w)$ with $1<p<\infty$ and $w \in$ $A_{p}$. For arbitrary $x_{0} \in \mathbb{R}^{n}$, set $B=B\left(x_{0}, r_{B}\right)$ for the ball centered at $x_{0}$ and of radius $r_{B}$. We represent $f_{j}$ as

$$
f_{j}=f_{j} \cdot \chi_{2 B}+f_{j} \cdot \chi_{(2 B)^{c}}:=f_{j}^{0}+f_{j}^{\infty},
$$

where $\chi_{2 B}$ denotes the characteristic function of $2 B=B\left(x_{0}, 2 r_{B}\right) \subseteq \mathbb{R}^{n}, j=$ $1,2, \ldots$. Then we write

$$
\begin{aligned}
& \frac{1}{\theta(w(B))^{1 / p}}\left(\int_{B}\left(\sum_{j}\left|\mathcal{S}_{\alpha}\left(f_{j}\right)(x)\right|^{2}\right)^{p / 2} w(x) d x\right)^{1 / p} \\
\leq & \frac{1}{\theta(w(B))^{1 / p}}\left(\int_{B}\left(\sum_{j}\left|\mathcal{S}_{\alpha}\left(f_{j}^{0}\right)(x)\right|^{2}\right)^{p / 2} w(x) d x\right)^{1 / p} \\
& +\frac{1}{\theta(w(B))^{1 / p}}\left(\int_{B}\left(\sum_{j}\left|\mathcal{S}_{\alpha}\left(f_{j}^{\infty}\right)(x)\right|^{2}\right)^{p / 2} w(x) d x\right)^{1 / p} \\
:= & I_{1}+I_{2} .
\end{aligned}
$$

Let us first estimate $I_{1}$. From the boundedness of vector-valued intrinsic square functions in $L_{w}^{p}\left(\mathbb{R}^{n}\right)$ (see Theorem A), it follows that

$$
\begin{aligned}
I_{1} & \leq \frac{1}{\theta(w(B))^{1 / p}}\left\|\left(\sum_{j}\left|\mathcal{S}_{\alpha}\left(f_{j}^{0}\right)\right|^{2}\right)^{1 / 2}\right\|_{L_{w}^{p}} \\
& \leq C \cdot \frac{1}{\theta(w(B))^{1 / p}}\left(\int_{2 B}\left(\sum_{j}\left|f_{j}(x)\right|^{2}\right)^{p / 2} w(x) d x\right)^{1 / p} \\
& \leq C\left\|\left(\sum_{j}\left|f_{j}\right|^{2}\right)^{1 / 2}\right\|_{\mathcal{M}^{p, \theta}(w)} \cdot \frac{\theta(w(2 B))^{1 / p}}{\theta(w(B))^{1 / p}} .
\end{aligned}
$$

Moreover, since $0<w(B)<w(2 B)<+\infty$ when $w \in A_{p}$ with $1<p<\infty$, then by the $\mathcal{D}_{\kappa}$ condition (2.9) of $\theta$ and the inequality (2.1), we obtain

$$
\begin{aligned}
I_{1} & \leq C\left\|\left(\sum_{j}\left|f_{j}\right|^{2}\right)^{1 / 2}\right\|_{\mathcal{M}^{p, \theta}(w)} \cdot \frac{w(2 B)^{\kappa / p}}{w(B)^{\kappa / p}} \\
& \leq C\left\|\left(\sum_{j}\left|f_{j}\right|^{2}\right)^{1 / 2}\right\|_{\mathcal{M}^{p, \theta}(w)} \cdot
\end{aligned}
$$


We now consider the other term $I_{2}$. For any $\varphi \in \mathcal{C}_{\alpha}, 0<\alpha \leq 1, j=1,2, \ldots$, and $(y, t) \in \Gamma(x)$ with $x \in B$, we have

$$
\begin{aligned}
\left|\int_{\mathbb{R}^{n}} \varphi_{t}(y-z) f_{j}^{\infty}(z) d z\right| & =\left|\int_{(2 B)^{c}} \varphi_{t}(y-z) f_{j}(z) d z\right| \\
& \leq C \cdot t^{-n} \int_{(2 B)^{c} \cap\{z:|y-z| \leq t\}}\left|f_{j}(z)\right| d z \\
& \leq C \cdot t^{-n} \sum_{l=1}^{\infty} \int_{\left(2^{l+1} B \backslash 2^{l} B\right) \cap\{z:|y-z| \leq t\}}\left|f_{j}(z)\right| d z .
\end{aligned}
$$

Since $|y-z| \leq t$ and $(y, t) \in \Gamma(x)$, then one has $|x-z| \leq|x-y|+|y-z| \leq 2 t$. Hence, for any $x \in B$ and $z \in\left(2^{l+1} B \backslash 2^{l} B\right)$, a direct computation shows that

$$
2 t \geq|x-z| \geq\left|z-x_{0}\right|-\left|x-x_{0}\right| \geq 2^{l-1} r_{B} .
$$

Therefore, by using the above inequalities (3.1) and (3.2) together with Minkowski's inequality for integrals, we can deduce

$$
\begin{aligned}
\mathcal{S}_{\alpha}\left(f_{j}^{\infty}\right)(x) & =\left(\iint_{\Gamma(x)} \sup _{\varphi \in \mathcal{C}_{\alpha}}\left|\int_{\mathbb{R}^{n}} \varphi_{t}(y-z) f_{j}^{\infty}(z) d z\right|^{2} \frac{d y d t}{t^{n+1}}\right)^{1 / 2} \\
& \leq C\left(\int_{2^{l-2} r_{B}}^{\infty} \int_{|x-y|<t}\left|t^{-n} \sum_{l=1}^{\infty} \int_{2^{l+1} B \backslash 2^{l} B}\right| f_{j}(z)|d z|^{2} \frac{d y d t}{t^{n+1}}\right)^{1 / 2} \\
& \leq C\left(\sum_{l=1}^{\infty} \int_{2^{l+1} B \backslash 2^{l} B}\left|f_{j}(z)\right| d z\right)\left(\int_{2^{l-2} r_{B}}^{\infty} \frac{d t}{t^{2 n+1}}\right)^{1 / 2} \\
& \leq C \sum_{l=1}^{\infty} \frac{1}{\left|2^{l+1} B\right|} \int_{2^{l+1} B \backslash 2^{l} B}\left|f_{j}(z)\right| d z .
\end{aligned}
$$

Then by duality and Cauchy-Schwarz inequality, we get

$$
\begin{aligned}
& \left(\sum_{j}\left|\mathcal{S}_{\alpha}\left(f_{j}^{\infty}\right)(x)\right|^{2}\right)^{1 / 2} \\
& \leq C\left(\sum_{j}\left|\sum_{l=1}^{\infty} \frac{1}{\left|2^{l+1} B\right|} \int_{2^{l+1} B \backslash 2^{l} B}\right| f_{j}(z)|d z|^{2}\right)^{1 / 2} \\
& \leq C \sup _{\left(\sum_{j}\left|\zeta_{j}\right|^{2}\right)^{1 / 2} \leq 1} \sum_{j}\left(\sum_{l=1}^{\infty} \frac{1}{\left|2^{l+1} B\right|} \int_{2^{l+1} B}\left|f_{j}(z)\right| d z \cdot \zeta_{j}\right) \\
& \leq C \sum_{l=1}^{\infty} \frac{1}{\left|2^{l+1} B\right|} \int_{2^{l+1} B} \sup _{\left(\sum_{j}\left|\zeta_{j}\right|^{2}\right)^{1 / 2} \leq 1}\left(\sum_{j}\left|f_{j}(z)\right| \cdot \zeta_{j}\right) d z \\
& \leq C \sum_{l=1}^{\infty} \frac{1}{\left|2^{l+1} B\right|} \int_{2^{l+1} B}\left(\sum_{j}\left|f_{j}(z)\right|^{2}\right)^{1 / 2} d z .
\end{aligned}
$$


Furthermore, it follows from Hölder's inequality and $A_{p}$ condition on $w$ that

$$
\begin{aligned}
& \left(\sum_{j}\left|\mathcal{S}_{\alpha}\left(f_{j}^{\infty}\right)(x)\right|^{2}\right)^{1 / 2} \\
& \leq C \sum_{l=1}^{\infty} \frac{1}{\left|2^{l+1} B\right|}\left(\int_{2^{l+1} B}\left(\sum_{j}\left|f_{j}(z)\right|^{2}\right)^{p / 2} w(z) d z\right)^{1 / p} \times\left(\int_{2^{l+1} B} w(z)^{-p^{\prime} / p} d z\right)^{1 / p^{\prime}} \\
& \leq C\left\|\left(\sum_{j}\left|f_{j}\right|^{2}\right)^{1 / 2}\right\|_{\mathcal{M}^{p, \theta}(w)} \times \sum_{l=1}^{\infty} \frac{\theta\left(w\left(2^{l+1} B\right)\right)^{1 / p}}{w\left(2^{l+1} B\right)^{1 / p}} .
\end{aligned}
$$

Hence, by the above pointwise estimate,

$$
I_{2} \leq C\left\|\left(\sum_{j}\left|f_{j}\right|^{2}\right)^{1 / 2}\right\|_{\mathcal{M}^{p, \theta}(w)} \times \sum_{l=1}^{\infty} \frac{\theta\left(w\left(2^{l+1} B\right)\right)^{1 / p}}{\theta(w(B))^{1 / p}} \cdot \frac{w(B)^{1 / p}}{w\left(2^{l+1} B\right)^{1 / p}} .
$$

For any $l \in \mathbb{Z}^{+}$, since $0<w(B)<w\left(2^{l+1} B\right)<+\infty$ when $w \in A_{p} \subset A_{\infty}$ with $1<p<\infty$, then by using the $\mathcal{D}_{\kappa}$ condition (2.9) of $\theta$ again, the inequality (2.3) with exponent $\delta>0$ and the fact that $0 \leq \kappa<1$, we find that

$$
\begin{aligned}
\sum_{l=1}^{\infty} \frac{\theta\left(w\left(2^{l+1} B\right)\right)^{1 / p}}{\theta(w(B))^{1 / p}} \cdot \frac{w(B)^{1 / p}}{w\left(2^{l+1} B\right)^{1 / p}} & \leq C \sum_{l=1}^{\infty} \frac{w(B)^{(1-\kappa) / p}}{w\left(2^{l+1} B\right)^{(1-\kappa) / p}} \\
& \leq C \sum_{l=1}^{\infty}\left(\frac{|B|}{\left|2^{l+1} B\right|}\right)^{\delta(1-\kappa) / p} \\
& \leq C \sum_{l=1}^{\infty}\left(\frac{1}{2^{(l+1) n}}\right)^{\delta(1-\kappa) / p} \\
& \leq C,
\end{aligned}
$$

where the last series is convergent since the exponent $\delta(1-\kappa) / p$ is positive. This implies our desired estimate

$$
I_{2} \leq C\left\|\left(\sum_{j}\left|f_{j}\right|^{2}\right)^{1 / 2}\right\|_{\mathcal{M}^{p, \theta}(w)} .
$$

Combining the above two estimates for $I_{1}$ and $I_{2}$, and then taking the supremum over all balls $B \subset \mathbb{R}^{n}$, we complete the proof of Theorem 2.1]

Proof of Theorem 2.2. Let $\left(\sum_{j}\left|f_{j}\right|^{2}\right)^{1 / 2} \in \mathcal{M}^{1, \theta}(w)$ with $w \in A_{1}$. For arbitrary $x_{0} \in \mathbb{R}^{n}$, set $B=B\left(x_{0}, r_{B}\right)$ for the ball centered at $x_{0}$ and of radius $r_{B}$. Write $f_{j}=f_{j}^{0}+f_{j}^{\infty}$ with $f_{j}^{0}=f_{j} \cdot \chi_{2 B}$ and $f_{j}^{\infty}=f_{j} \cdot \chi_{(2 B)^{c}}, j=1,2, \ldots$ Then for any given $\sigma>0$, we have

$$
\frac{1}{\theta(w(B))} \sigma \cdot w\left(\left\{x \in B:\left(\sum_{j}\left|\mathcal{S}_{\alpha}\left(f_{j}\right)(x)\right|^{2}\right)^{1 / 2}>\sigma\right\}\right)
$$




$$
\begin{aligned}
& \quad \leq \frac{1}{\theta(w(B))} \sigma \cdot w\left(\left\{x \in B:\left(\sum_{j}\left|\mathcal{S}_{\alpha}\left(f_{j}^{0}\right)(x)\right|^{2}\right)^{1 / 2}>\sigma / 2\right\}\right) \\
& \quad+\frac{1}{\theta(w(B))} \sigma \cdot w\left(\left\{x \in B:\left(\sum_{j}\left|\mathcal{S}_{\alpha}\left(f_{j}^{\infty}\right)(x)\right|^{2}\right)^{1 / 2}>\sigma / 2\right\}\right) \\
& :=I_{1}^{\prime}+I_{2}^{\prime} .
\end{aligned}
$$

Below we will give the estimates of $I_{1}^{\prime}$ and $I_{2}^{\prime}$, respectively. From the weighted weak $(1,1)$ boundedness of vector-valued intrinsic square functions (see Theorem B), it follows that

$$
\begin{aligned}
I_{1}^{\prime} & \leq \frac{2}{\theta(w(B))}\left\|\left(\sum_{j}\left|\mathcal{S}_{\alpha}\left(f_{j}^{0}\right)\right|^{2}\right)^{1 / 2}\right\|_{W L_{w}^{1}} \\
& \leq C \cdot \frac{1}{\theta(w(B))}\left(\int_{2 B}\left(\sum_{j}\left|f_{j}(x)\right|^{2}\right)^{1 / 2} w(x) d x\right) \\
& \leq C\left\|\left(\sum_{j}\left|f_{j}\right|^{2}\right)^{1 / 2}\right\|_{\mathcal{M}^{1, \theta}(w)} \cdot \frac{\theta(w(2 B))}{\theta(w(B))} .
\end{aligned}
$$

Moreover, since $0<w(B)<w(2 B)<+\infty$ when $w \in A_{1}$, then by the $\mathcal{D}_{\kappa}$ condition (2.9) of $\theta$ and inequality (2.1), we get

$$
\begin{aligned}
I_{1}^{\prime} & \leq C\left\|\left(\sum_{j}\left|f_{j}\right|^{2}\right)^{1 / 2}\right\|_{\mathcal{M}^{1, \theta}(w)} \cdot \frac{w(2 B)^{\kappa}}{w(B)^{\kappa}} \\
& \leq C\left\|\left(\sum_{j}\left|f_{j}\right|^{2}\right)^{1 / 2}\right\|_{\mathcal{M}^{1, \theta}(w)} .
\end{aligned}
$$

As for the term $I_{2}^{\prime}$, it follows directly from Chebyshev's inequality and the pointwise estimate (3.3) that

$$
\begin{aligned}
I_{2}^{\prime} & \leq \frac{1}{\theta(w(B))} \sigma \cdot \frac{2}{\sigma} \int_{B}\left(\sum_{j}\left|\mathcal{S}_{\alpha}\left(f_{j}^{\infty}\right)(x)\right|^{2}\right)^{1 / 2} w(x) d x \\
& \leq C \cdot \frac{w(B)}{\theta(w(B))} \sum_{l=1}^{\infty} \frac{1}{\left|2^{l+1} B\right|} \int_{2^{l+1} B}\left(\sum_{j}\left|f_{j}(z)\right|^{2}\right)^{1 / 2} d z .
\end{aligned}
$$

Another application of $A_{1}$ condition on $w$ gives that

$$
\begin{aligned}
& \frac{1}{\left|2^{l+1} B\right|} \int_{2^{l+1} B}\left(\sum_{j}\left|f_{j}(z)\right|^{2}\right)^{1 / 2} d z \\
& \leq C \frac{1}{w\left(2^{l+1} B\right)} \cdot \underset{z \in 2^{l+1} B}{\operatorname{essinf}} w(z) \int_{2^{l+1} B}\left(\sum_{j}\left|f_{j}(z)\right|^{2}\right)^{1 / 2} d z \\
& \leq C \frac{1}{w\left(2^{l+1} B\right)}\left(\int_{2^{l+1} B}\left(\sum_{j}\left|f_{j}(z)\right|^{2}\right)^{1 / 2} w(z) d z\right)
\end{aligned}
$$




$$
\leq C\left\|\left(\sum_{j}\left|f_{j}\right|^{2}\right)^{1 / 2}\right\|_{\mathcal{M}^{1, \theta}(w)} \cdot \frac{\theta\left(w\left(2^{l+1} B\right)\right)}{w\left(2^{l+1} B\right)} .
$$

Substituting the above inequality (3.6) into (3.5), we thus obtain

$$
I_{2}^{\prime} \leq C\left\|\left(\sum_{j}\left|f_{j}\right|^{2}\right)^{1 / 2}\right\|_{\mathcal{M}^{1, \theta}(w)} \times \sum_{l=1}^{\infty} \frac{\theta\left(w\left(2^{l+1} B\right)\right)}{\theta(w(B))} \cdot \frac{w(B)}{w\left(2^{l+1} B\right)} .
$$

Note that $w \in A_{1} \subset A_{\infty}$, then one has $0<w(B)<w\left(2^{l+1} B\right)<+\infty$ for any $l \in \mathbb{Z}^{+}$. Thus, by using the $\mathcal{D}_{\kappa}$ condition (2.9) of $\theta$ again, the inequality (2.3) with exponent $\delta^{*}>0$ and the fact that $0 \leq \kappa<1$, we find that

$$
\begin{aligned}
\sum_{l=1}^{\infty} \frac{\theta\left(w\left(2^{l+1} B\right)\right)}{\theta(w(B))} \cdot \frac{w(B)}{w\left(2^{l+1} B\right)} & \leq C \sum_{l=1}^{\infty} \frac{w(B)^{1-\kappa}}{w\left(2^{l+1} B\right)^{1-\kappa}} \\
& \leq C \sum_{l=1}^{\infty}\left(\frac{|B|}{\left|2^{l+1} B\right|}\right)^{\delta^{*}(1-\kappa)} \\
& \leq C \sum_{l=1}^{\infty}\left(\frac{1}{2^{(l+1) n}}\right)^{\delta^{*}(1-\kappa)} \\
& \leq C .
\end{aligned}
$$

Therefore,

$$
I_{2}^{\prime} \leq C\left\|\left(\sum_{j}\left|f_{j}\right|^{2}\right)^{1 / 2}\right\|_{\mathcal{M}^{1, \theta}(w)} .
$$

Summing up the above estimates for $I_{1}^{\prime}$ and $I_{2}^{\prime}$, and then taking the supremum over all balls $B \subset \mathbb{R}^{n}$ and all $\sigma>0$, we finish the proof of Theorem 2.2.

\section{Proof of Theorem 2.3}

Given a real-valued function $b \in B M O\left(\mathbb{R}^{n}\right)$, we will follow the idea developed in [1,2] and denote $F(\xi)=e^{\xi[b(x)-b(z)]}, \xi \in \mathbb{C}$. Then by the analyticity of $F(\xi)$ on $\mathbb{C}$ and the Cauchy integral formula, we get

$$
\begin{aligned}
b(x)-b(z) & =F^{\prime}(0)=\frac{1}{2 \pi i} \int_{|\xi|=1} \frac{F(\xi)}{\xi^{2}} d \xi \\
& =\frac{1}{2 \pi} \int_{0}^{2 \pi} e^{e^{i \theta}[b(x)-b(z)]} \cdot e^{-i \theta} d \theta .
\end{aligned}
$$

Thus, for any $\varphi \in \mathcal{C}_{\alpha}, 0<\alpha \leq 1$ and $j \in \mathbb{Z}^{+}$, we obtain

$$
\begin{aligned}
& \left|\int_{\mathbb{R}^{n}}[b(x)-b(z)] \varphi_{t}(y-z) f_{j}(z) d z\right| \\
= & \left|\frac{1}{2 \pi} \int_{0}^{2 \pi}\left(\int_{\mathbb{R}^{n}} \varphi_{t}(y-z) e^{-e^{i \theta} b(z)} f_{j}(z) d z\right) e^{e^{i \theta} b(x)} \cdot e^{-i \theta} d \theta\right|
\end{aligned}
$$




$$
\begin{aligned}
& \leq \frac{1}{2 \pi} \int_{0}^{2 \pi} \sup _{\varphi \in \mathcal{C}_{\alpha}}\left|\int_{\mathbb{R}^{n}} \varphi_{t}(y-z) e^{-e^{i \theta} b(z)} f_{j}(z) d z\right| e^{\cos \theta \cdot b(x)} d \theta \\
& \leq \frac{1}{2 \pi} \int_{0}^{2 \pi} A_{\alpha}\left(e^{-e^{i \theta} b} \cdot f_{j}\right)(y, t) \cdot e^{\cos \theta \cdot b(x)} d \theta .
\end{aligned}
$$

So we have

$$
\left|\left[b, \mathcal{S}_{\alpha}\right]\left(f_{j}\right)(x)\right| \leq \frac{1}{2 \pi} \int_{0}^{2 \pi} \mathcal{S}_{\alpha}\left(e^{-e^{i \theta} b} \cdot f_{j}\right)(x) \cdot e^{\cos \theta \cdot b(x)} d \theta .
$$

Moreover, by using standard duality argument and Cauchy-Schwarz inequality, we get

$$
\begin{aligned}
& \left(\sum_{j}\left|\left[b, \mathcal{S}_{\alpha}\right]\left(f_{j}\right)(x)\right|^{2}\right)^{1 / 2} \\
& \leq \frac{1}{2 \pi}\left(\sum_{j}\left|\int_{0}^{2 \pi} \mathcal{S}_{\alpha}\left(e^{-e^{i \theta} b} \cdot f_{j}\right)(x) \cdot e^{\cos \theta \cdot b(x)} d \theta\right|^{2}\right)^{1 / 2} \\
& \leq \frac{1}{2 \pi} \sup _{\left(\sum_{j}\left|\zeta_{j}\right|^{2}\right)^{1 / 2} \leq 1} \sum_{j}\left(\int_{0}^{2 \pi} \mathcal{S}_{\alpha}\left(e^{-e^{i \theta} b} \cdot f_{j}\right)(x) \cdot e^{\cos \theta \cdot b(x)} d \theta \cdot \zeta_{j}\right) \\
& \leq \frac{1}{2 \pi} \int_{0}^{2 \pi} \sup _{\left(\sum_{j}\left|\zeta_{j}\right|^{2}\right)^{1 / 2} \leq 1}\left(\sum_{j} \mathcal{S}_{\alpha}\left(e^{-e^{i \theta} b} \cdot f_{j}\right)(x) \cdot e^{\cos \theta \cdot b(x)} \cdot \zeta_{j}\right) d \theta \\
& \leq \frac{1}{2 \pi} \int_{0}^{2 \pi}\left(\sum_{j}\left|\mathcal{S}_{\alpha}\left(e^{-e^{i \theta} b} \cdot f_{j}\right)(x)\right|^{2}\right)^{1 / 2} \cdot e^{\cos \theta \cdot b(x)} d \theta .
\end{aligned}
$$

Therefore, by the $L_{w}^{p}$-boundedness of vector-valued intrinsic square functions (see Theorem A), and using the same arguments as in 2], we can also show the following result.

Theorem 4.1. Let $0<\alpha \leq 1,1<p<\infty$ and $w \in A_{p}$. Then there exists a constant $C>0$ independent of $\vec{f}=\left(f_{1}, f_{2}, \ldots\right)$ such that

$$
\left\|\left(\sum_{j}\left|\left[b, \mathcal{S}_{\alpha}\right]\left(f_{j}\right)\right|^{2}\right)^{1 / 2}\right\|_{L_{w}^{p}} \leq C\left\|\left(\sum_{j}\left|f_{j}\right|^{2}\right)^{1 / 2}\right\|_{L_{w}^{p}}
$$

provided that $b \in B M O\left(\mathbb{R}^{n}\right)$.

We are now in a position to give the proof of Theorem 2.3 .

Proof. Let $\left(\sum_{j}\left|f_{j}\right|^{2}\right)^{1 / 2} \in \mathcal{M}^{p, \theta}(w)$ with $1<p<\infty$ and $w \in A_{p}$. Fix $x_{0} \in \mathbb{R}^{n}$ and let $B=B\left(x_{0}, r_{B}\right)$ be a ball centered at $x_{0}$ of radius $r_{B}$. We split $f_{j}$ by $f_{j}=f_{j}^{0}+f_{j}^{\infty}$, where $f_{j}^{0}=f_{j} \cdot \chi_{2 B}$ and $2 B=B\left(x_{0}, 2 r_{B}\right) \subseteq \mathbb{R}^{n}, j=1,2, \ldots$. Then we write

$$
\frac{1}{\theta(w(B))^{1 / p}}\left(\int_{B}\left(\sum_{j}\left|\left[b, \mathcal{S}_{\alpha}\right]\left(f_{j}\right)(x)\right|^{2}\right)^{p / 2} w(x) d x\right)^{1 / p}
$$




$$
\begin{aligned}
\leq & \frac{1}{\theta(w(B))^{1 / p}}\left(\int_{B}\left(\sum_{j}\left|\left[b, \mathcal{S}_{\alpha}\right]\left(f_{j}^{0}\right)(x)\right|^{2}\right)^{p / 2} w(x) d x\right)^{1 / p} \\
& +\frac{1}{\theta(w(B))^{1 / p}}\left(\int_{B}\left(\sum_{j}\left|\left[b, \mathcal{S}_{\alpha}\right]\left(f_{j}^{\infty}\right)(x)\right|^{2}\right)^{p / 2} w(x) d x\right)^{1 / p} \\
:= & J_{1}+J_{2} .
\end{aligned}
$$

By using Theorem 4.1, the $\mathcal{D}_{\kappa}$ condition (2.9) of $\theta$ and the inequality (2.1), we obtain

$$
\begin{aligned}
J_{1} & \leq \frac{1}{\theta(w(B))^{1 / p}}\left\|\left(\sum_{j}\left|\left[b, \mathcal{S}_{\alpha}\right]\left(f_{j}^{0}\right)\right|^{2}\right)^{1 / 2}\right\|_{L_{w}^{p}} \\
& \leq C \cdot \frac{1}{\theta(w(B))^{1 / p}}\left(\int_{2 B}\left(\sum_{j}\left|f_{j}(x)\right|^{2}\right)^{p / 2} w(x) d x\right)^{1 / p} \\
& \leq C\left\|\left(\sum_{j}\left|f_{j}\right|^{2}\right)^{1 / 2}\right\|_{\mathcal{M}^{p, \theta}(w)} \cdot \frac{\theta(w(2 B))^{1 / p}}{\theta(w(B))^{1 / p}} \\
& \leq C\left\|\left(\sum_{j}\left|f_{j}\right|^{2}\right)^{1 / 2}\right\|_{\mathcal{M}^{p, \theta}(w)} \cdot \frac{w(2 B)^{\kappa / p}}{w(B)^{\kappa / p}} \\
& \leq C\left\|\left(\sum_{j}\left|f_{j}\right|^{2}\right)^{1 / 2}\right\|_{\mathcal{M}^{p, \theta}(w)} \cdot
\end{aligned}
$$

Let us now turn to estimate the other term $J_{2}$. For any given $x \in B,(y, t) \in \Gamma(x)$ and for $j=1,2, \ldots$, we have

$$
\begin{aligned}
\sup _{\varphi \in \mathcal{C}_{\alpha}}\left|\int_{\mathbb{R}^{n}}[b(x)-b(z)] \varphi_{t}(y-z) f_{j}^{\infty}(z) d z\right| & \leq\left|b(x)-b_{B}\right| \cdot \sup _{\varphi \in \mathcal{C}_{\alpha}}\left|\int_{\mathbb{R}^{n}} \varphi_{t}(y-z) f_{j}^{\infty}(z) d z\right| \\
& +\sup _{\varphi \in \mathcal{C}_{\alpha}}\left|\int_{\mathbb{R}^{n}}\left[b_{B}-b(z)\right] \varphi_{t}(y-z) f_{j}^{\infty}(z) d z\right| .
\end{aligned}
$$

By definition, we thus have

$$
\left|\left[b, \mathcal{S}_{\alpha}\right]\left(f_{j}^{\infty}\right)(x)\right| \leq\left|b(x)-b_{B}\right| \cdot \mathcal{S}_{\alpha}\left(f_{j}^{\infty}\right)(x)+\mathcal{S}_{\alpha}\left(\left[b_{B}-b\right] f_{j}^{\infty}\right)(x) .
$$

From this and Minkowski' inequality for series, we further obtain

$$
\begin{aligned}
\left(\sum_{j}\left|\left[b, \mathcal{S}_{\alpha}\right]\left(f_{j}^{\infty}\right)(x)\right|^{2}\right)^{1 / 2} & \leq\left|b(x)-b_{B}\right|\left(\sum_{j}\left|\mathcal{S}_{\alpha}\left(f_{j}^{\infty}\right)(x)\right|^{2}\right)^{1 / 2} \\
& +\left(\sum_{j}\left|\mathcal{S}_{\alpha}\left(\left[b_{B}-b\right] f_{j}^{\infty}\right)(x)\right|^{2}\right)^{1 / 2}
\end{aligned}
$$


For any $\varphi \in \mathcal{C}_{\alpha}, 0<\alpha \leq 1, j=1,2, \ldots$, and $(y, t) \in \Gamma(x)$ with $x \in B$, we have

$$
\begin{aligned}
& \left|\int_{\mathbb{R}^{n}}\left[b_{B}-b(z)\right] \varphi_{t}(y-z) f_{j}^{\infty}(z) d z\right| \\
& =\left|\int_{(2 B)^{c}}\left[b_{B}-b(z)\right] \varphi_{t}(y-z) f_{j}(z) d z\right| \\
& \leq C \cdot t^{-n} \int_{(2 B)^{c} \cap\{z:|y-z| \leq t\}}\left|b(z)-b_{B}\right|\left|f_{j}(z)\right| d z \\
& \leq C \cdot t^{-n} \sum_{l=1}^{\infty} \int_{\left(2^{l+1} B \backslash 2^{l} B\right) \cap\{z:|y-z| \leq t\}}\left|b(z)-b_{B}\right|\left|f_{j}(z)\right| d z .
\end{aligned}
$$

Hence, for any $x \in B$, by using the inequalities (4.1) and (3.2) together with Minkowski's inequality for integrals, we can deduce that

$$
\begin{aligned}
& \mathcal{S}_{\alpha}\left(\left[b_{B}-b\right] f_{j}^{\infty}\right)(x) \\
& =\left(\iint_{\Gamma(x)} \sup _{\varphi \in \mathcal{C}_{\alpha}}\left|\int_{\mathbb{R}^{n}}\left[b_{B}-b(z)\right] \varphi_{t}(y-z) f_{j}^{\infty}(z) d z\right|^{2} \frac{d y d t}{t^{n+1}}\right)^{1 / 2} \\
& \leq C\left(\int_{2^{l-2} r_{B}}^{\infty} \int_{|x-y|<t}\left|t^{-n} \sum_{l=1}^{\infty} \int_{2^{l+1} B \backslash 2^{l} B}\right| b(z)-b_{B}|| f_{j}(z)|d z|^{2} \frac{d y d t}{t^{n+1}}\right)^{1 / 2} \\
& \leq C\left(\sum_{l=1}^{\infty} \int_{2^{l+1} B \backslash 2^{l} B}\left|b(z)-b_{B}\right|\left|f_{j}(z)\right| d z\right)\left(\int_{2^{l-2} r_{B}}^{\infty} \frac{d t}{t^{2 n+1}}\right)^{1 / 2} \\
& \leq C \sum_{l=1}^{\infty} \frac{1}{\left|2^{l+1} B\right|} \int_{2^{l+1} B \backslash 2^{l} B}\left|b(z)-b_{B}\right|\left|f_{j}(z)\right| d z .
\end{aligned}
$$

Therefore, by duality and Cauchy-Schwarz inequality, we get

$$
\begin{aligned}
& \left(\sum_{j}\left|\mathcal{S}_{\alpha}\left(\left[b_{B}-b\right] f_{j}^{\infty}\right)(x)\right|^{2}\right)^{1 / 2} \\
& \leq C\left(\sum_{j}\left|\sum_{l=1}^{\infty} \frac{1}{\left|2^{l+1} B\right|} \int_{2^{l+1} B \backslash 2^{l} B}\right| b(z)-b_{B}|| f_{j}(z)|d z|^{2}\right)^{1 / 2} \\
& \leq C \sup _{\left(\sum_{j}\left|\zeta_{j}\right|^{2}\right)^{1 / 2} \leq 1} \sum_{j}\left(\sum_{l=1}^{\infty} \frac{1}{\left|2^{l+1} B\right|} \int_{2^{l+1} B}\left|b(z)-b_{B}\right|\left|f_{j}(z)\right| d z \cdot \zeta_{j}\right) \\
& \leq C \sum_{l=1}^{\infty} \frac{1}{\left|2^{l+1} B\right|} \int_{2^{l+1} B} \sup _{j}\left(\left.\sum_{j}\right|^{2}\right)^{1 / 2} \leq 1 \\
& \left.\leq C \sum_{l=1}^{\infty} \frac{1}{\left|2^{l+1} B\right|} \int_{2^{l+1} B}\left|b(z)-b_{B}\right|\left|f_{j}(z)\right| \cdot \zeta_{j}\right) d z
\end{aligned}
$$


Consequently, from the pointwise estimates (3.3) and (4.2), it follows that

$$
\begin{aligned}
J_{2} & \leq \frac{C}{\theta(w(B))^{1 / p}}\left(\int_{B}\left|b(x)-b_{B}\right|^{p} w(x) d x\right)^{1 / p} \times\left(\sum_{l=1}^{\infty} \frac{1}{\left|2^{l+1} B\right|} \int_{2^{l+1} B}\left(\sum_{j}\left|f_{j}(z)\right|^{2}\right)^{1 / 2} d z\right) \\
& +C \cdot \frac{w(B)^{1 / p}}{\theta(w(B))^{1 / p}} \sum_{l=1}^{\infty} \frac{1}{\left|2^{l+1} B\right|} \int_{2^{l+1} B}\left|b_{2^{l+1} B}-b_{B}\right|\left(\sum_{j}\left|f_{j}(z)\right|^{2}\right)^{1 / 2} d z \\
& +C \cdot \frac{w(B)^{1 / p}}{\theta(w(B))^{1 / p}} \sum_{l=1}^{\infty} \frac{1}{\left|2^{l+1} B\right|} \int_{2^{l+1} B}\left|b(z)-b_{2^{l+1} B}\right|\left(\sum_{j}\left|f_{j}(z)\right|^{2}\right)^{1 / 2} d z \\
& :=J_{3}+J_{4}+J_{5} .
\end{aligned}
$$

Recall that the following estimate holds for $w \in A_{p}$ and $1 \leq p<\infty$ :

$$
\left(\int_{B}\left|b(x)-b_{B}\right|^{p} w(x) d x\right)^{1 / p} \leq C\|b\|_{*} \cdot w(B)^{1 / p} .
$$

Indeed, since $w \in A_{p}$ with $1 \leq p<\infty$, we know that there exists a number $r>1$ such that $w \in R H_{r}$. By using Hölder's inequality and John-Nirenberg's inequality for $B M O$ functions (see [3], 5$]$ ), we find that

$$
\begin{aligned}
\left(\int_{B}\left|b(x)-b_{B}\right|^{p} w(x) d x\right)^{1 / p} & \leq\left(\int_{B}\left|b(x)-b_{B}\right|^{p r^{\prime}} d x\right)^{1 /\left(p r^{\prime}\right)}\left(\int_{B} w(x)^{r} d x\right)^{1 /(p r)} \\
& \leq C \cdot w(B)^{1 / p}\left(\frac{1}{|B|} \int_{B}\left|b(x)-b_{B}\right|^{p r^{\prime}} d x\right)^{1 /\left(p r^{\prime}\right)} \\
& \leq C\|b\|_{*} \cdot w(B)^{1 / p} .
\end{aligned}
$$

Furthermore, it follows from (4.6), Hölder's inequality and the $A_{p}$ condition on $w$ that

$$
\begin{aligned}
J_{3} \leq & C\|b\|_{*} \cdot \frac{w(B)^{1 / p}}{\theta(w(B))^{1 / p}} \sum_{l=1}^{\infty} \frac{1}{\left|2^{l+1} B\right|}\left(\int_{2^{l+1} B}\left(\sum_{j}\left|f_{j}(z)\right|^{2}\right)^{p / 2} w(z) d z\right)^{1 / p} \\
& \times\left(\int_{2^{l+1} B} w(z)^{-p^{\prime} / p} d z\right)^{1 / p^{\prime}} \\
& \leq C\left\|\left(\sum_{j}\left|f_{j}\right|^{2}\right)^{1 / 2}\right\|_{\mathcal{M}^{p, \theta}(w)} \times \sum_{l=1}^{\infty} \frac{\theta\left(w\left(2^{l+1} B\right)\right)^{1 / p}}{\theta(w(B))^{1 / p}} \cdot \frac{w(B)^{1 / p}}{w\left(2^{l+1} B\right)^{1 / p}} \\
& \leq C\left\|\left(\sum_{j}\left|f_{j}\right|^{2}\right)^{1 / 2}\right\|_{\mathcal{M}^{p, \theta}(w)},
\end{aligned}
$$

where in the last inequality we have used the estimate (3.4). For the term $J_{4}$, since $b \in B M O\left(\mathbb{R}^{n}\right)$, a simple calculation shows that for every ball $B$ (or cube Q)

$$
\left|b_{2^{l+1} B}-b_{B}\right| \leq C \cdot(l+1)\|b\|_{*} .
$$


We then use Hölder's inequality, (4.4) and the $A_{p}$ condition on $w$ to obtain

$$
\begin{aligned}
J_{4} \leq & C\|b\|_{*} \cdot \frac{w(B)^{1 / p}}{\theta(w(B))^{1 / p}} \sum_{l=1}^{\infty} \frac{l+1}{\left|2^{l+1} B\right|}\left(\int_{2^{l+1} B}\left(\sum_{j}\left|f_{j}(z)\right|^{2}\right)^{p / 2} w(z) d z\right)^{1 / p} \\
& \times\left(\int_{2^{l+1} B} w(z)^{-p^{\prime} / p} d z\right)^{1 / p^{\prime}} \\
& \leq C\left\|\left(\sum_{j}\left|f_{j}\right|^{2}\right)^{1 / 2}\right\|_{\mathcal{M}^{p, \theta}(w)} \times \sum_{l=1}^{\infty}(l+1) \cdot \frac{\theta\left(w\left(2^{l+1} B\right)\right)^{1 / p}}{\theta(w(B))^{1 / p}} \cdot \frac{w(B)^{1 / p}}{w\left(2^{l+1} B\right)^{1 / p}} .
\end{aligned}
$$

Applying the $\mathcal{D}_{\kappa}$ condition (2.9) of $\theta$ and the inequality (2.3) again together with the fact that $0 \leq \kappa<1$, we thus have

$$
\begin{aligned}
\sum_{l=1}^{\infty}(l+1) \cdot \frac{\theta\left(w\left(2^{l+1} B\right)\right)^{1 / p}}{\theta(w(B))^{1 / p}} \cdot \frac{w(B)^{1 / p}}{w\left(2^{l+1} B\right)^{1 / p}} & \leq C \sum_{l=1}^{\infty}(l+1) \cdot \frac{w(B)^{(1-\kappa) / p}}{w\left(2^{l+1} B\right)^{(1-\kappa) / p}} \\
& \leq C \sum_{l=1}^{\infty}(l+1) \cdot\left(\frac{|B|}{\left|2^{l+1} B\right|}\right)^{\delta(1-\kappa) / p} \\
& \leq C \sum_{l=1}^{\infty}(l+1) \cdot\left(\frac{1}{2^{(l+1) n}}\right)^{\delta(1-\kappa) / p} \\
& \leq C
\end{aligned}
$$

which in turn gives that

$$
J_{4} \leq C\left\|\left(\sum_{j}\left|f_{j}\right|^{2}\right)^{1 / 2}\right\|_{\mathcal{M}^{p, \theta}(w)} .
$$

It remains to estimate the last term $J_{5}$. An application of Hölder's inequality gives us that

$$
\begin{aligned}
J_{5} \leq & C \cdot \frac{w(B)^{1 / p}}{\theta(w(B))^{1 / p}} \sum_{l=1}^{\infty} \frac{1}{\left|2^{l+1} B\right|}\left(\int_{2^{l+1} B}\left(\sum_{j}\left|f_{j}(z)\right|^{2}\right)^{p / 2} w(z) d z\right)^{1 / p} \\
& \times\left(\int_{2^{l+1} B}\left|b(z)-b_{2^{l+1} B}\right|^{p^{\prime}} w(z)^{-p^{\prime} / p} d z\right)^{1 / p^{\prime}} .
\end{aligned}
$$

If we set $\mu(z)=w(z)^{-p^{\prime} / p}$, then we have $\mu \in A_{p^{\prime}}$ because $w \in A_{p}$ (see [3, 4]). Then it follows from the inequality (4.6) and the $A_{p}$ condition that

$$
\begin{aligned}
\left(\int_{2^{l+1} B}\left|b(z)-b_{2^{l+1} B}\right|^{p^{\prime}} \mu(z) d z\right)^{1 / p^{\prime}} & \leq C\|b\|_{*} \cdot \mu\left(2^{l+1} B\right)^{1 / p^{\prime}} \\
& =C\|b\|_{*} \cdot\left(\int_{2^{l+1} B} w(z)^{-p^{\prime} / p} d z\right)^{1 / p^{\prime}} \\
& \leq C\|b\|_{*} \cdot \frac{\left|2^{l+1} B\right|}{w\left(2^{l+1} B\right)^{1 / p}}
\end{aligned}
$$


Therefore, in view of the estimates (4.6) and (3.4), we conclude that

$$
\begin{aligned}
J_{5} & \leq C\|b\|_{*} \cdot \frac{w(B)^{1 / p}}{\theta(w(B))^{1 / p}} \sum_{l=1}^{\infty} \frac{1}{w\left(2^{l+1} B\right)^{1 / p}}\left(\int_{2^{l+1} B}\left(\sum_{j}\left|f_{j}(z)\right|^{2}\right)^{p / 2} w(z) d z\right)^{1 / p} \\
& \leq C\left\|\left(\sum_{j}\left|f_{j}\right|^{2}\right)^{1 / 2}\right\| \|_{\mathcal{M}^{p, \theta}(w)} \times \sum_{l=1}^{\infty} \frac{\theta\left(w\left(2^{l+1} B\right)\right)^{1 / p}}{\theta(w(B))^{1 / p}} \cdot \frac{w(B)^{1 / p}}{w\left(2^{l+1} B\right)^{1 / p}} \\
& \leq C\left\|\left(\sum_{j}\left|f_{j}\right|^{2}\right)^{1 / 2}\right\|_{\mathcal{M}^{p, \theta}(w)} .
\end{aligned}
$$

Summarizing the above discussions, we finish the proof of the main theorem.

\section{Proof of Theorem 2.4}

Proof. Inspired by the works in 11, 13, 20, for any fixed $\sigma>0$, we apply the Calderón-Zygmund decomposition of $\vec{f}=\left(f_{1}, f_{2}, \ldots\right)$ at height $\sigma$ to obtain a collection of disjoint non-overlapping dyadic cubes $\left\{Q_{i}\right\}$ such that the following property holds (see [13,15])

$$
\sigma<\frac{1}{\left|Q_{i}\right|} \int_{Q_{i}}\left(\sum_{j}\left|f_{j}(y)\right|^{2}\right)^{1 / 2} d y \leq 2^{n} \cdot \sigma,
$$

where $Q_{i}=Q\left(c_{i}, \ell_{i}\right)$ denotes the cube centered at $c_{i}$ with side length $\ell_{i}$ and all cubes are assumed to have their sides parallel to the coordinate axes. If we set $E=\bigcup_{i} Q_{i}$, then

$$
\left(\sum_{j}\left|f_{j}(y)\right|^{2}\right)^{1 / 2} \leq \sigma, \quad \text { a.e. } x \in \mathbb{R}^{n} \backslash E .
$$

Now we proceed to construct vector-valued version of the Calderón-Zygmund decomposition. Define two vector-valued functions $\vec{g}=\left(g_{1}, g_{2}, \ldots\right)$ and $\vec{h}=$ $\left(h_{1}, h_{2}, \ldots\right)$ as follows:

$$
g_{j}(x)= \begin{cases}f_{j}(x) & \text { if } x \in E^{c}, \\ \frac{1}{\left|Q_{i}\right|} \int_{Q_{i}} f_{j}(y) d y & \text { if } x \in Q_{i},\end{cases}
$$

and

$$
h_{j}(x)=f_{j}(x)-g_{j}(x)=\sum_{i} h_{i j}(x), \quad j=1,2, \ldots,
$$

where $h_{i j}(x)=h_{j}(x) \cdot \chi_{Q_{i}}(x)=\left(f_{j}(x)-g_{j}(x)\right) \cdot \chi_{Q_{i}}(x)$. Then we have

$$
\left(\sum_{j}\left|g_{j}(x)\right|^{2}\right)^{1 / 2} \leq C \cdot \sigma, \quad \text { a.e. } x \in \mathbb{R}^{n}
$$


and

$$
\vec{f}=\vec{g}+\vec{h}:=\left(g_{1}+h_{1}, g_{2}+h_{2}, \ldots\right) .
$$

Obviously, $h_{i j}$ is supported on $Q_{i}, i, j=1,2, \ldots$,

$$
\int_{\mathbb{R}^{n}} h_{i j}(x) d x=0, \quad \text { and } \quad\left\|h_{i j}\right\|_{L^{1}}=\int_{\mathbb{R}^{n}}\left|h_{i j}(x)\right| d x \leq 2 \int_{Q_{i}}\left|f_{j}(x)\right| d x
$$

according to the above decomposition. By (5.3) and Minkowski's inequality,

$$
\left(\sum_{j}\left|\left[b, \mathcal{S}_{\alpha}\right]\left(f_{j}\right)(x)\right|^{2}\right)^{1 / 2} \leq\left(\sum_{j}\left|\left[b, \mathcal{S}_{\alpha}\right]\left(g_{j}\right)(x)\right|^{2}\right)^{1 / 2}+\left(\sum_{j}\left|\left[b, \mathcal{S}_{\alpha}\right]\left(h_{j}\right)(x)\right|^{2}\right)^{1 / 2} \text {. }
$$

Then we can write

$$
\begin{aligned}
& w\left(\left\{x \in \mathbb{R}^{n}:\left(\sum_{j}\left|\left[b, \mathcal{S}_{\alpha}\right]\left(f_{j}\right)(x)\right|^{2}\right)^{1 / 2}>\sigma\right\}\right) \\
& \leq w\left(\left\{x \in \mathbb{R}^{n}:\left(\sum_{j}\left|\left[b, \mathcal{S}_{\alpha}\right]\left(g_{j}\right)(x)\right|^{2}\right)^{1 / 2}>\sigma / 2\right\}\right) \\
& +w\left(\left\{x \in \mathbb{R}^{n}:\left(\sum_{j}\left|\left[b, \mathcal{S}_{\alpha}\right]\left(h_{j}\right)(x)\right|^{2}\right)^{1 / 2}>\sigma / 2\right\}\right) \\
& :=K_{1}+K_{2} .
\end{aligned}
$$

Observe that $w \in A_{1} \subset A_{2}$. Applying Chebyshev's inequality and Theorem 4.1 we obtain

$$
K_{1} \leq \frac{4}{\sigma^{2}} \cdot\left\|\left(\sum_{j}\left|\left[b, \mathcal{S}_{\alpha}\right]\left(g_{j}\right)\right|^{2}\right)^{1 / 2}\right\|_{L_{w}^{2}}^{2} \leq \frac{C}{\sigma^{2}} \cdot\left\|\left(\sum_{j}\left|g_{j}\right|^{2}\right)^{1 / 2}\right\|_{L_{w}^{2}}^{2} .
$$

Moreover, by the inequality (5.2),

$$
\begin{aligned}
& \left\|\left(\sum_{j}\left|g_{j}\right|^{2}\right)^{1 / 2}\right\|_{L_{w}^{2}}^{2} \\
& \leq C \cdot \sigma \int_{\mathbb{R}^{n}}\left(\sum_{j}\left|g_{j}(x)\right|^{2}\right)^{1 / 2} w(x) d x \\
& \leq C \cdot \sigma\left(\int_{E^{c}}\left(\sum_{j}\left|f_{j}(x)\right|^{2}\right)^{1 / 2} w(x) d x+\int_{\cup_{i} Q_{i}}\left(\sum_{j}\left|g_{j}(x)\right|^{2}\right)^{1 / 2} w(x) d x\right) .
\end{aligned}
$$

Recall that $g_{j}(x)=\frac{1}{\left|Q_{i}\right|} \int_{Q_{i}} f_{j}(y) d y$ when $x \in Q_{i}$. As before, by using duality and Cauchy-Schwarz inequality, we can see the following estimate is valid for all $x \in Q_{i}$.

$$
\left(\sum_{j}\left|g_{j}(x)\right|^{2}\right)^{1 / 2} \leq \frac{1}{\left|Q_{i}\right|} \int_{Q_{i}}\left(\sum_{j}\left|f_{j}(y)\right|^{2}\right)^{1 / 2} d y
$$


This estimate (5.4) along with the $A_{1}$ condition yields

$$
\begin{aligned}
& \left\|\left(\sum_{j}\left|g_{j}\right|^{2}\right)^{1 / 2}\right\|_{L_{w}^{2}}^{2} \\
& \leq C \cdot \sigma\left(\int_{\mathbb{R}^{n}}\left(\sum_{j}\left|f_{j}(x)\right|^{2}\right)^{1 / 2} w(x) d x+\sum_{i} \frac{w\left(Q_{i}\right)}{\left|Q_{i}\right|} \int_{Q_{i}}\left(\sum_{j}\left|f_{j}(y)\right|^{2}\right)^{1 / 2} d y\right) \\
& \leq C \cdot \sigma\left(\int_{\mathbb{R}^{n}}\left(\sum_{j}\left|f_{j}(x)\right|^{2}\right)^{1 / 2} w(x) d x+\sum_{i}{\underset{y \in Q}{y s i n f}}_{y \in Q_{i}} w(y) \int_{Q_{i}}\left(\sum_{j}\left|f_{j}(y)\right|^{2}\right)^{1 / 2} d y\right) \\
& \leq C \cdot \sigma\left(\int_{\mathbb{R}^{n}}\left(\sum_{j}\left|f_{j}(x)\right|^{2}\right)^{1 / 2} w(x) d x+\int_{\bigcup_{i} Q_{i}}\left(\sum_{j}\left|f_{j}(y)\right|^{2}\right)^{1 / 2} w(y) d y\right) \\
& \leq C \cdot \sigma \int_{\mathbb{R}^{n}}\left(\sum_{j}\left|f_{j}(x)\right|^{2}\right)^{1 / 2} w(x) d x .
\end{aligned}
$$

So we have

$$
K_{1} \leq C \int_{\mathbb{R}^{n}} \frac{\|\vec{f}(x)\|_{\ell^{2}}}{\sigma} \cdot w(x) d x \leq C \int_{\mathbb{R}^{n}} \Phi\left(\frac{\|\vec{f}(x)\|_{\ell^{2}}}{\sigma}\right) \cdot w(x) d x .
$$

To deal with the other term $K_{2}$, let $Q_{i}^{*}=2 \sqrt{n} Q_{i}$ be the cube concentric with $Q_{i}$ such that $\ell\left(Q_{i}^{*}\right)=(2 \sqrt{n}) \ell\left(Q_{i}\right)$. Then we can further decompose $K_{2}$ as follows.

$$
\begin{aligned}
K_{2} \leq & w\left(\left\{x \in \bigcup_{i} Q_{i}^{*}:\left(\sum_{j}\left|\left[b, \mathcal{S}_{\alpha}\right]\left(h_{j}\right)(x)\right|^{2}\right)^{1 / 2}>\sigma / 2\right\}\right) \\
& +w\left(\left\{x \notin \bigcup_{i} Q_{i}^{*}:\left(\sum_{j}\left|\left[b, \mathcal{S}_{\alpha}\right]\left(h_{j}\right)(x)\right|^{2}\right)^{1 / 2}>\sigma / 2\right\}\right) \\
:= & K_{3}+K_{4} .
\end{aligned}
$$

Since $w \in A_{1}$, then by the inequality (2.1), we can get

$$
K_{3} \leq \sum_{i} w\left(Q_{i}^{*}\right) \leq C \sum_{i} w\left(Q_{i}\right) .
$$

Furthermore, it follows from the inequality (5.1) and the $A_{1}$ condition that

$$
\begin{aligned}
K_{3} & \leq C \sum_{i} \frac{1}{\sigma} \cdot \underset{y \in Q_{i}}{\operatorname{essinf}} w(y) \int_{Q_{i}}\left(\sum_{j}\left|f_{j}(y)\right|^{2}\right)^{1 / 2} d y \\
& \leq \frac{C}{\sigma} \sum_{i} \int_{Q_{i}}\left(\sum_{j}\left|f_{j}(y)\right|^{2}\right)^{1 / 2} w(y) d y \\
& \leq \frac{C}{\sigma} \int_{\bigcup_{i} Q_{i}}\left(\sum_{j}\left|f_{j}(y)\right|^{2}\right)^{1 / 2} w(y) d y
\end{aligned}
$$




$$
\leq C \int_{\mathbb{R}^{n}} \frac{\|\vec{f}(y)\|_{\ell^{2}}}{\sigma} \cdot w(y) d y \leq C \int_{\mathbb{R}^{n}} \Phi\left(\frac{\|\vec{f}(y)\|_{\ell^{2}}}{\sigma}\right) \cdot w(y) d y .
$$

Arguing as in the proof of Theorem 2.3. for any given $x \in \mathbb{R}^{n},(y, t) \in \Gamma(x)$ and for $j=1,2, \ldots$, we also find that

$$
\begin{aligned}
& \sup _{\varphi \in \mathcal{C}_{\alpha}}\left|\int_{\mathbb{R}^{n}}[b(x)-b(z)] \varphi_{t}(y-z) \sum_{i} h_{i j}(z) d z\right| \\
& \leq \sup _{\varphi \in \mathcal{C}_{\alpha}}\left|\sum_{i}\left[b(x)-b_{Q_{i}}\right] \int_{\mathbb{R}^{n}} \varphi_{t}(y-z) h_{i j}(z) d z\right| \\
& +\sup _{\varphi \in \mathcal{C}_{\alpha}}\left|\int_{\mathbb{R}^{n}} \varphi_{t}(y-z) \sum_{i}\left[b_{Q_{i}}-b(z)\right] h_{i j}(z) d z\right| \\
& \leq \sum_{i}\left|b(x)-b_{Q_{i}}\right| \cdot \sup _{\varphi \in \mathcal{C}_{\alpha}}\left|\int_{\mathbb{R}^{n}} \varphi_{t}(y-z) h_{i j}(z) d z\right| \\
& +\sup _{\varphi \in \mathcal{C}_{\alpha}}\left|\int_{\mathbb{R}^{n}} \varphi_{t}(y-z) \sum_{i}\left[b_{Q_{i}}-b(z)\right] h_{i j}(z) d z\right| .
\end{aligned}
$$

Hence, by definition, we have that for any given $x \in \mathbb{R}^{n}$ and $j \in \mathbb{Z}^{+}$,

$$
\left|\left[b, \mathcal{S}_{\alpha}\right]\left(h_{j}\right)(x)\right| \leq \sum_{i}\left|b(x)-b_{Q_{i}}\right| \cdot \mathcal{S}_{\alpha}\left(h_{i j}\right)(x)+\mathcal{S}_{\alpha}\left(\sum_{i}\left[b_{Q_{i}}-b\right] h_{i j}\right)(x) .
$$

On the other hand, by duality argument and Cauchy-Schwarz inequality, we can see the following vector-valued form of Minkowski's inequality is true for any real numbers $\nu_{i j} \in \mathbb{R}, i, j=1,2, \ldots$.

$$
\left(\sum_{j}\left|\sum_{i}\right| \nu_{i j}||^{2}\right)^{1 / 2} \leq \sum_{i}\left(\sum_{j}\left|\nu_{i j}\right|^{2}\right)^{1 / 2} .
$$

Therefore, by the estimates (5.6) and (5.7), we get

$$
\begin{aligned}
\left(\sum_{j}\left|\left[b, \mathcal{S}_{\alpha}\right]\left(h_{j}\right)(x)\right|^{2}\right)^{1 / 2} & \leq\left(\sum_{j}\left|\sum_{i}\right| b(x)-b_{Q_{i}}\left|\cdot \mathcal{S}_{\alpha}\left(h_{i j}\right)(x)\right|^{2}\right)^{1 / 2} \\
& +\left(\sum_{j}\left|\mathcal{S}_{\alpha}\left(\sum_{i}\left[b_{Q_{i}}-b\right] h_{i j}\right)(x)\right|^{2}\right)^{1 / 2} \\
& \leq \sum_{i}\left|b(x)-b_{Q_{i}}\right| \cdot\left(\sum_{j}\left|\mathcal{S}_{\alpha}\left(h_{i j}\right)(x)\right|^{2}\right)^{1 / 2} \\
& +\left(\sum_{j}\left|\mathcal{S}_{\alpha}\left(\sum_{i}\left[b_{Q_{i}}-b\right] h_{i j}\right)(x)\right|^{2}\right)^{1 / 2} .
\end{aligned}
$$


Then the term $K_{4}$ can be divided into two parts.

$$
\begin{aligned}
K_{4} \leq & w\left(\left\{x \notin \bigcup_{i} Q_{i}^{*}: \sum_{i}\left|b(x)-b_{Q_{i}}\right| \cdot\left(\sum_{j}\left|\mathcal{S}_{\alpha}\left(h_{i j}\right)(x)\right|^{2}\right)^{1 / 2}>\sigma / 4\right\}\right) \\
& +w\left(\left\{x \notin \bigcup_{i} Q_{i}^{*}:\left(\sum_{j}\left|\mathcal{S}_{\alpha}\left(\sum_{i}\left[b_{Q_{i}}-b\right] h_{i j}\right)(x)\right|^{2}\right)^{1 / 2}>\sigma / 4\right\}\right) \\
:= & K_{5}+K_{6} .
\end{aligned}
$$

It follows directly from the Chebyshev's inequality that

$$
\begin{aligned}
K_{5} & \leq \frac{4}{\sigma} \int_{\mathbb{R}^{n} \backslash \bigcup_{i} Q_{i}^{*}} \sum_{i}\left|b(x)-b_{Q_{i}}\right| \cdot\left(\sum_{j}\left|\mathcal{S}_{\alpha}\left(h_{i j}\right)(x)\right|^{2}\right)^{1 / 2} w(x) d x \\
& \leq \frac{4}{\sigma} \sum_{i}\left(\int_{\left(Q_{i}^{*}\right)^{c}}\left|b(x)-b_{Q_{i}}\right| \cdot\left(\sum_{j}\left|\mathcal{S}_{\alpha}\left(h_{i j}\right)(x)\right|^{2}\right)^{1 / 2} w(x) d x\right) .
\end{aligned}
$$

Denote by $c_{i}$ the center of $Q_{i}$. For any $\varphi \in \mathcal{C}_{\alpha}, 0<\alpha \leq 1$, by the cancellation condition of $h_{i j}$ over $Q_{i}$, we obtain that for any $(y, t) \in \Gamma(x)$ and for $i, j=$ $1,2, \ldots$,

$$
\begin{aligned}
\left|\left(\varphi_{t} * h_{i j}\right)(y)\right| & =\left|\int_{Q_{i}}\left[\varphi_{t}(y-z)-\varphi_{t}\left(y-c_{i}\right)\right] h_{i j}(z) d z\right| \\
& \leq \int_{Q_{i} \cap\{z:|z-y| \leq t\}} \frac{\left|z-c_{i}\right|^{\alpha}}{t^{n+\alpha}}\left|h_{i j}(z)\right| d z \\
& \leq C \cdot \frac{\ell\left(Q_{i}\right)^{\alpha}}{t^{n+\alpha}} \int_{Q_{i} \cap\{z:|z-y| \leq t\}}\left|h_{i j}(z)\right| d z .
\end{aligned}
$$

In addition, for any $z \in Q_{i}$ and $x \in\left(Q_{i}^{*}\right)^{c}$, we have $\left|z-c_{i}\right|<\frac{\left|x-c_{i}\right|}{2}$. Thus, for all $(y, t) \in \Gamma(x)$ and $|z-y| \leq t$ with $z \in Q_{i}$, it is easy to see that

$$
t+t \geq|x-y|+|y-z| \geq|x-z| \geq\left|x-c_{i}\right|-\left|z-c_{i}\right| \geq \frac{\left|x-c_{i}\right|}{2} .
$$

Hence, for any $x \in\left(Q_{i}^{*}\right)^{c}$, by using the above inequalities (5.8) and (5.9) along with the fact that $\left\|h_{i j}\right\|_{L^{1}} \leq 2 \int_{Q_{i}}\left|f_{j}(x)\right| d x$, we obtain that for any $i, j=$ $1,2, \ldots$,

$$
\begin{aligned}
\left|\mathcal{S}_{\alpha}\left(h_{i j}\right)(x)\right| & =\left(\iint_{\Gamma(x)}\left(\sup _{\varphi \in \mathcal{C}_{\alpha}}\left|\left(\varphi_{t} * h_{i j}\right)(y)\right|\right)^{2} \frac{d y d t}{t^{n+1}}\right)^{1 / 2} \\
& \leq C \cdot \ell\left(Q_{i}\right)^{\alpha}\left(\int_{Q_{i}}\left|h_{i j}(z)\right| d z\right)\left(\int_{\frac{\left|x-c_{i}\right|}{4}}^{\infty} \int_{|y-x|<t} \frac{d y d t}{t^{2(n+\alpha)+n+1}}\right)^{1 / 2} \\
& \leq C \cdot \ell\left(Q_{i}\right)^{\alpha}\left(\int_{Q_{i}}\left|h_{i j}(z)\right| d z\right)\left(\int_{\frac{\left|x-c_{i}\right|}{4}}^{\infty} \frac{d t}{t^{2(n+\alpha)+1}}\right)^{1 / 2}
\end{aligned}
$$




$$
\leq C \cdot \frac{\ell\left(Q_{i}\right)^{\alpha}}{\left|x-c_{i}\right|^{n+\alpha}}\left(\int_{Q_{i}}\left|f_{j}(z)\right| d z\right) .
$$

Furthermore, by duality and Cauchy-Schwarz inequality again,

$$
\left(\sum_{j}\left|\mathcal{S}_{\alpha}\left(h_{i j}\right)(x)\right|^{2}\right)^{1 / 2} \leq C \cdot \frac{\ell\left(Q_{i}\right)^{\alpha}}{\left|x-c_{i}\right|^{n+\alpha}} \times \int_{Q_{i}}\left(\sum_{j}\left|f_{j}(z)\right|^{2}\right)^{1 / 2} d z .
$$

Since $Q_{i}^{*}=2 \sqrt{n} Q_{i} \supset 2 Q_{i}$, then $\left(Q_{i}^{*}\right)^{c} \subset\left(2 Q_{i}\right)^{c}$. This fact together with the above pointwise estimate yields

$$
\begin{aligned}
K_{5} & \leq \frac{C}{\sigma} \sum_{i}\left(\ell\left(Q_{i}\right)^{\alpha} \int_{Q_{i}}\left(\sum_{j}\left|f_{j}(z)\right|^{2}\right)^{1 / 2} d z \times \int_{\left(Q_{i}^{*}\right)^{c}}\left|b(x)-b_{Q_{i}}\right| \cdot \frac{w(x)}{\left|x-c_{i}\right|^{n+\alpha}} d x\right) \\
& \leq \frac{C}{\sigma} \sum_{i}\left(\ell\left(Q_{i}\right)^{\alpha} \int_{Q_{i}}\left(\sum_{j}\left|f_{j}(z)\right|^{2}\right)^{1 / 2} d z \times \int_{\left(2 Q_{i}\right)^{c}}\left|b(x)-b_{Q_{i}}\right| \cdot \frac{w(x)}{\left|x-c_{i}\right|^{n+\alpha}} d x\right) \\
& \leq \frac{C}{\sigma} \sum_{i}\left(\ell\left(Q_{i}\right)^{\alpha} \int_{Q_{i}}\left(\sum_{j}\left|f_{j}(z)\right|^{2}\right)^{1 / 2} d z \times \sum_{l=1}^{\infty} \int_{2^{l+1} Q_{i} \backslash 2^{l} Q_{i}}\left|b(x)-b_{2^{l+1} Q_{i}}\right| \cdot \frac{w(x)}{\left|x-c_{i}\right|^{n+\alpha}} d x\right) \\
& +\frac{C}{\sigma} \sum_{i}\left(\ell\left(Q_{i}\right)^{\alpha} \int_{Q_{i}}\left(\sum_{j}\left|f_{j}(z)\right|^{2}\right)^{1 / 2} d z \times \sum_{l=1}^{\infty} \int_{2^{l+1} Q_{i} \backslash 2^{l} Q_{i}}\left|b_{2^{l+1} Q_{i}}-b_{Q_{i}}\right| \cdot \frac{w(x)}{\left|x-c_{i}\right|^{n+\alpha}} d x\right) \\
& :=\mathrm{I}+\mathrm{II} .
\end{aligned}
$$

For the term I, it then follows from (4.6) (consider $2^{l+1} Q_{i}$ instead of $B$ ), (2.2) and the fact that $w \in A_{1}$,

$$
\begin{aligned}
\mathrm{I} & \leq \frac{C}{\sigma} \sum_{i}\left(\ell\left(Q_{i}\right)^{\alpha} \int_{Q_{i}}\left(\sum_{j}\left|f_{j}(z)\right|^{2}\right)^{1 / 2} d z \times \sum_{l=1}^{\infty} \frac{1}{\left[2^{l-1} \ell\left(Q_{i}\right)\right]^{n+\alpha}} \int_{2^{l+1} Q_{i}}\left|b(x)-b_{2^{l+1} Q_{i}}\right| \cdot w(x) d x\right) \\
& \leq \frac{C \cdot\|b\|_{*}}{\sigma} \sum_{i}\left(\int_{Q_{i}}\left(\sum_{j}\left|f_{j}(z)\right|^{2}\right)^{1 / 2} d z \times \sum_{l=1}^{\infty} \frac{w\left(2^{l+1} Q_{i}\right)}{\left(2^{l-1}\right)^{n+\alpha}\left|Q_{i}\right|}\right) \\
& \leq \frac{C \cdot\|b\|_{*}}{\sigma} \sum_{i}\left(\int_{Q_{i}}\left(\sum_{j}\left|f_{j}(z)\right|^{2}\right)^{1 / 2} d z \times \sum_{l=1}^{\infty} \frac{\left(2^{l+1}\right)^{n} w\left(Q_{i}\right)}{\left(2^{l-1}\right)^{n+\alpha}\left|Q_{i}\right|}\right) \\
& \leq \frac{C}{\sigma} \sum_{i}\left(\frac{w\left(Q_{i}\right)}{\left|Q_{i}\right|} \cdot \int_{Q_{i}}\left(\sum_{j}\left|f_{j}(z)\right|^{2}\right)^{1 / 2} d z \times \sum_{l=1}^{\infty} \frac{1}{2^{l \alpha}}\right) \\
& \leq \frac{C}{\sigma} \sum_{i} \underset{z \in Q_{i}}{\operatorname{essinf} w(z)} \int_{Q_{i}}\left(\sum_{j}\left|f_{j}(z)\right|^{2}\right)^{1 / 2} d z
\end{aligned}
$$




$$
\begin{aligned}
& \leq \frac{C}{\sigma} \int_{\bigcup_{i} Q_{i}}\left(\sum_{j}\left|f_{j}(z)\right|^{2}\right)^{1 / 2} w(z) d z \\
& \leq C \int_{\mathbb{R}^{n}} \frac{\|\vec{f}(z)\|_{\ell^{2}}}{\sigma} \cdot w(z) d z \leq C \int_{\mathbb{R}^{n}} \Phi\left(\frac{\|\vec{f}(z)\|_{\ell^{2}}}{\sigma}\right) \cdot w(z) d z .
\end{aligned}
$$

For the term II, from the inequalities (4.4) and (2.2) along with the fact that $w \in A_{1}$, it then follows that

$$
\begin{aligned}
\mathrm{II} & \leq \frac{C \cdot\|b\|_{*}}{\sigma} \sum_{i}\left(\ell\left(Q_{i}\right)^{\alpha} \int_{Q_{i}}\left(\sum_{j}\left|f_{j}(z)\right|^{2}\right)^{1 / 2} d z \times \sum_{l=1}^{\infty}(l+1) \cdot \frac{w\left(2^{l+1} Q_{i}\right)}{\left[2^{l-1} \ell\left(Q_{i}\right)\right]^{n+\alpha}}\right) \\
& \leq \frac{C \cdot\|b\|_{*}}{\sigma} \sum_{i}\left(\int_{Q_{i}}\left(\sum_{j}\left|f_{j}(z)\right|^{2}\right)^{1 / 2} d z \times \sum_{l=1}^{\infty}(l+1) \cdot \frac{\left(2^{l+1}\right)^{n} w\left(Q_{i}\right)}{\left(2^{l-1}\right)^{n+\alpha}\left|Q_{i}\right|}\right) \\
& \leq \frac{C}{\sigma} \sum_{i}\left(\frac{w\left(Q_{i}\right)}{\left|Q_{i}\right|} \cdot \int_{Q_{i}}\left(\sum_{j}\left|f_{j}(z)\right|^{2}\right)^{1 / 2} d z \times \sum_{l=1}^{\infty} \frac{l+1}{2^{l \alpha}}\right) \\
& \leq \frac{C}{\sigma} \sum_{i}\left(\frac{w\left(Q_{i}\right)}{\left|Q_{i}\right|} \cdot \int_{Q_{i}}\left(\sum_{j}\left|f_{j}(z)\right|^{2}\right)^{1 / 2} d z\right) \leq C \int_{\mathbb{R}^{n}} \Phi\left(\frac{\|\vec{f}(z)\|_{\ell^{2}}}{\sigma}\right) \cdot w(z) d z .
\end{aligned}
$$

On the other hand, by using the weighted weak-type $(1,1)$ estimate of vectorvalued intrinsic square functions (see Theorem B) and (5.7), we have

$$
\begin{aligned}
K_{6} & \leq \frac{C}{\sigma} \int_{\mathbb{R}^{n}}\left(\sum_{j}\left|\sum_{i}\right| b(x)-b_{Q_{i}}|| h_{i j}(x)||^{2}\right)^{1 / 2} w(x) d x \\
& \leq \frac{C}{\sigma} \int_{\mathbb{R}^{n}} \sum_{i}\left|b(x)-b_{Q_{i}}\right|\left(\sum_{j}\left|h_{i j}(x)\right|^{2}\right)^{1 / 2} w(x) d x \\
& =\frac{C}{\sigma} \sum_{i} \int_{Q_{i}}\left|b(x)-b_{Q_{i}}\right|\left(\sum_{j}\left|h_{i j}(x)\right|^{2}\right)^{1 / 2} w(x) d x \\
& \leq \frac{C}{\sigma} \sum_{i} \int_{Q_{i}}\left|b(x)-b_{Q_{i}}\right|\left(\sum_{j}\left|f_{j}(x)\right|^{2}\right)^{1 / 2} w(x) d x \\
& +\frac{C}{\sigma} \sum_{i} \frac{1}{\left|Q_{i}\right|} \int_{Q_{i}}\left(\sum_{j}\left|f_{j}(y)\right|^{2}\right)^{1 / 2} d y \times \int_{Q_{i}}\left|b(x)-b_{Q_{i}}\right| w(x) d x \\
& :=\mathrm{III}+\mathrm{IV} .
\end{aligned}
$$

For the term III, by the generalized Hölder's inequality with weight (2.6), (2.7) and (2.5), we can deduce that

$$
\mathrm{III}=\frac{C}{\sigma} \sum_{i} w\left(Q_{i}\right) \cdot \frac{1}{w\left(Q_{i}\right)} \int_{Q_{i}}\left|b(x)-b_{Q_{i}}\right|\left(\sum_{j}\left|f_{j}(x)\right|^{2}\right)^{1 / 2} w(x) d x
$$




$$
\begin{aligned}
& \leq \frac{C}{\sigma} \sum_{i} w\left(Q_{i}\right) \cdot\left\|b-b_{Q_{i}}\right\|_{\exp L(w), Q_{i}}\left\|\left(\sum_{j}\left|f_{j}\right|^{2}\right)^{1 / 2}\right\|_{L \log L(w), Q_{i}} \\
& \leq \frac{C \cdot\|b\|_{*}}{\sigma} \sum_{i} w\left(Q_{i}\right) \cdot\left\|\left(\sum_{j}\left|f_{j}\right|^{2}\right)^{1 / 2}\right\|_{L \log L(w), Q_{i}} \\
& \leq \frac{C \cdot\|b\|_{*}}{\sigma} \sum_{i} w\left(Q_{i}\right) \cdot \inf _{\eta>0}\left\{\eta+\frac{\eta}{w\left(Q_{i}\right)} \int_{Q_{i}} \Phi\left(\frac{\|\vec{f}(y)\|_{\ell^{2}}}{\eta}\right) \cdot w(y) d y\right\} \\
& \leq \frac{C \cdot\|b\|_{*}}{\sigma} \sum_{i} w\left(Q_{i}\right) \cdot\left\{\sigma+\frac{\sigma}{w\left(Q_{i}\right)} \int_{Q_{i}} \Phi\left(\frac{\|\vec{f}(y)\|_{\ell^{2}}}{\sigma}\right) \cdot w(y) d y\right\} \\
& \leq C\left\{\sum_{i} w\left(Q_{i}\right)+\sum_{i} \int_{Q_{i}} \Phi\left(\frac{\|\vec{f}(y)\|_{\ell^{2}}}{\sigma}\right) \cdot w(y) d y\right\} \\
& \leq C \int_{\mathbb{R}^{n}} \Phi\left(\frac{\|\vec{f}(y)\|_{\ell^{2}}}{\sigma}\right) \cdot w(y) d y .
\end{aligned}
$$

For the term IV, by the inequality (4.6) (consider $Q_{i}$ instead of $B$ ) and the fact that $w \in A_{1}$, we conclude that

$$
\begin{aligned}
\mathrm{IV} & \leq \frac{C \cdot\|b\|_{*}}{\sigma} \sum_{i} \frac{w\left(Q_{i}\right)}{\left|Q_{i}\right|} \int_{Q_{i}}\left(\sum_{j}\left|f_{j}(y)\right|^{2}\right)^{1 / 2} d y \\
& \leq \frac{C \cdot\|b\|_{*}}{\sigma} \sum_{i} \int_{Q_{i}}\left(\sum_{j}\left|f_{j}(y)\right|^{2}\right)^{1 / 2} w(y) d y \\
& \leq \frac{C \cdot\|b\|_{*}}{\sigma} \int_{\bigcup_{i} Q_{i}}\left(\sum_{j}\left|f_{j}(y)\right|^{2}\right)^{1 / 2} w(y) d y \\
& \leq C \int_{\mathbb{R}^{n}} \frac{\|\vec{f}(y)\|_{\ell^{2}}}{\sigma} \cdot w(y) d y \leq C \int_{\mathbb{R}^{n}} \Phi\left(\frac{\|\vec{f}(y)\|_{\ell^{2}}}{\sigma}\right) \cdot w(y) d y .
\end{aligned}
$$

Summing up all the above estimates, we get the desired result.

\section{Proof of Theorem 2.5}

Proof. Fix a ball $B=B\left(x_{0}, r_{B}\right) \subseteq \mathbb{R}^{n}$ and decompose $f_{j}=f_{j}^{0}+f_{j}^{\infty}$, where $f_{j}^{0}=f_{j} \cdot \chi_{2 B}$ and $\chi_{2 B}$ denotes the characteristic function of $2 B=B\left(x_{0}, 2 r_{B}\right)$, $j=1,2, \ldots$. For any $0 \leq \kappa<1, w \in A_{1}$ and any given $\sigma>0$, we then write

$$
\begin{aligned}
& \frac{1}{\theta(w(B))} \cdot w\left(\left\{x \in B:\left(\sum_{j}\left|\left[b, \mathcal{S}_{\alpha}\right]\left(f_{j}\right)(x)\right|^{2}\right)^{1 / 2}>\sigma\right\}\right) \\
\leq & \frac{1}{\theta(w(B))} \cdot w\left(\left\{x \in B:\left(\sum_{j}\left|\left[b, \mathcal{S}_{\alpha}\right]\left(f_{j}^{0}\right)(x)\right|^{2}\right)^{1 / 2}>\sigma / 2\right\}\right)
\end{aligned}
$$




$$
\begin{aligned}
& +\frac{1}{\theta(w(B))} \cdot w\left(\left\{x \in B:\left(\sum_{j}\left|\left[b, \mathcal{S}_{\alpha}\right]\left(f_{j}^{\infty}\right)(x)\right|^{2}\right)^{1 / 2}>\sigma / 2\right\}\right) \\
:= & K_{1}^{\prime}+K_{2}^{\prime} .
\end{aligned}
$$

By using Theorem 2.4, we get

$$
\begin{aligned}
K_{1}^{\prime} & \leq C \cdot \frac{1}{\theta(w(B))} \int_{\mathbb{R}^{n}} \Phi\left(\frac{1}{\sigma}\left(\sum_{j}\left|f_{j}^{0}(x)\right|^{2}\right)^{1 / 2}\right) \cdot w(x) d x \\
& =C \cdot \frac{1}{\theta(w(B))} \int_{2 B} \Phi\left(\frac{1}{\sigma}\left(\sum_{j}\left|f_{j}(x)\right|^{2}\right)^{1 / 2}\right) \cdot w(x) d x \\
& =C \cdot \frac{\theta(w(2 B))}{\theta(w(B))} \cdot \frac{1}{\theta(w(2 B))} \int_{2 B} \Phi\left(\frac{1}{\sigma}\left(\sum_{j}\left|f_{j}(x)\right|^{2}\right)^{1 / 2}\right) \cdot w(x) d x .
\end{aligned}
$$

Observe that

$$
\frac{1}{\theta(w(B))} \leq \frac{1+\log ^{+}\left(\frac{w(B)}{\theta(w(B))}\right)}{\theta(w(B))}=\frac{\Phi\left(\frac{w(B)}{\theta(w(B))}\right)}{w(B)} .
$$

Moreover, since $0<w(B)<w(2 B)<+\infty$ when $w \in A_{1}$, then by the $\mathcal{D}_{\kappa}$ condition (2.9) of $\theta$, the inequality (2.1) and the fact (6.1), we have

$$
\begin{aligned}
K_{1}^{\prime} & \leq C \cdot \frac{w(2 B)^{\kappa}}{w(B)^{\kappa}} \cdot \frac{1}{\theta(w(2 B))} \int_{2 B} \Phi\left(\frac{\|\vec{f}(x)\|_{\ell^{2}}}{\sigma}\right) \cdot w(x) d x \\
& \leq C \cdot \sup _{B}\left\{\frac{1}{\theta(w(B))} \int_{B} \Phi\left(\frac{\|\vec{f}(x)\|_{\ell^{2}}}{\sigma}\right) \cdot w(x) d x\right\} \\
& \leq C \cdot \sup _{B}\left\{\frac{\Phi\left(\frac{w(B)}{\theta(w(B))}\right)}{w(B)} \int_{B} \Phi\left(\frac{\|\vec{f}(x)\|_{\ell^{2}}}{\sigma}\right) \cdot w(x) d x\right\} .
\end{aligned}
$$

Recall that the following pointwise estimate holds for any $x \in B$,

$$
\begin{aligned}
\left(\sum_{j}\left|\left[b, \mathcal{S}_{\alpha}\right]\left(f_{j}^{\infty}\right)(x)\right|^{2}\right)^{1 / 2} & \leq\left|b(x)-b_{B}\right|\left(\sum_{j}\left|\mathcal{S}_{\alpha}\left(f_{j}^{\infty}\right)(x)\right|^{2}\right)^{1 / 2} \\
& +\left(\sum_{j}\left|\mathcal{S}_{\alpha}\left(\left[b_{B}-b\right] f_{j}^{\infty}\right)(x)\right|^{2}\right)^{1 / 2}
\end{aligned}
$$

So we can divide the term $K_{2}^{\prime}$ into two parts.

$$
\begin{aligned}
K_{2}^{\prime} \leq & \frac{1}{\theta(w(B))} \cdot w\left(\left\{x \in B:\left|b(x)-b_{B}\right| \cdot\left(\sum_{j}\left|\mathcal{S}_{\alpha}\left(f_{j}^{\infty}\right)(x)\right|^{2}\right)^{1 / 2}>\sigma / 4\right\}\right) \\
& +\frac{1}{\theta(w(B))} \cdot w\left(\left\{x \in B:\left(\sum_{j}\left|\mathcal{S}_{\alpha}\left(\left[b_{B}-b\right] f_{j}^{\infty}\right)(x)\right|^{2}\right)^{1 / 2}>\sigma / 4\right\}\right) \\
:= & K_{3}^{\prime}+K_{4}^{\prime} .
\end{aligned}
$$


Since $w \in A_{1}$, then there exists a number $r>1$ such that $w \in R H_{r}$. Hence, by using the previous pointwise estimate (3.3), Chebyshev's inequality together with Hölder's inequality and John-Nirenberg's inequality (see [5]), we conclude that

$$
\begin{aligned}
K_{3}^{\prime} & \leq \frac{1}{\theta(w(B))} \cdot \frac{4}{\sigma} \int_{B}\left|b(x)-b_{B}\right| \cdot\left(\sum_{j}\left|\mathcal{S}_{\alpha}\left(f_{j}^{\infty}\right)(x)\right|^{2}\right)^{1 / 2} w(x) d x \\
& \leq C \sum_{l=1}^{\infty} \frac{1}{\left|2^{l+1} B\right|} \int_{2^{l+1} B} \frac{1}{\sigma}\left(\sum_{j}\left|f_{j}(z)\right|^{2}\right)^{1 / 2} d z \\
& \times \frac{1}{\theta(w(B))} \cdot\left(\int_{B}\left|b(x)-b_{B}\right|^{r^{\prime}} d x\right)^{1 / r^{\prime}}\left(\int_{B} w(x)^{r} d x\right)^{1 / r} \\
& \leq C \sum_{l=1}^{\infty} \frac{1}{\left|2^{l+1} B\right|} \int_{2^{l+1} B} \frac{\|\vec{f}(z)\|_{\ell^{2}}}{\sigma} d z \times \frac{w(B)}{\theta(w(B))} .
\end{aligned}
$$

It then follows from the $A_{1}$ condition and the fact (6.1) that

$$
\begin{aligned}
K_{3}^{\prime} & \leq C \sum_{l=1}^{\infty} \frac{1}{w\left(2^{l+1} B\right)} \int_{2^{l+1} B} \frac{\|\vec{f}(z)\|_{\ell^{2}}}{\sigma} \cdot w(z) d z \times \frac{w(B)}{\theta(w(B))} \\
& =C \sum_{l=1}^{\infty} \frac{1}{\theta\left(w\left(2^{l+1} B\right)\right)} \cdot \frac{\theta\left(w\left(2^{l+1} B\right)\right)}{w\left(2^{l+1} B\right)} \int_{2^{l+1} B} \frac{\|\vec{f}(z)\|_{\ell^{2}}}{\sigma} \cdot w(z) d z \times \frac{w(B)}{\theta(w(B))} \\
& \leq C \cdot \sup _{B}\left\{\frac{\Phi\left(\frac{w(B)}{\theta(w(B))}\right)}{w(B)} \int_{B} \Phi\left(\frac{\|\vec{f}(z)\|_{\ell^{2}}}{\sigma}\right) \cdot w(z) d z\right\} \\
& \times \sum_{l=1}^{\infty} \frac{\theta\left(w\left(2^{l+1} B\right)\right)}{\theta(w(B))} \cdot \frac{w(B)}{w\left(2^{l+1} B\right)} .
\end{aligned}
$$

Substituting the previous inequality (3.7) into the term $K_{3}^{\prime}$, we thus obtain

$$
K_{3}^{\prime} \leq C \cdot \sup _{B}\left\{\frac{\Phi\left(\frac{w(B)}{\theta(w(B))}\right)}{w(B)} \int_{B} \Phi\left(\frac{\|\vec{f}(z)\|_{\ell^{2}}}{\sigma}\right) \cdot w(z) d z\right\} .
$$

On the other hand, applying the previous pointwise estimate (4.2) and Chebyshev's inequality, we have

$$
\begin{aligned}
K_{4}^{\prime} & \leq \frac{1}{\theta(w(B))} \cdot \frac{4}{\sigma} \int_{B}\left(\sum_{j}\left|\mathcal{S}_{\alpha}\left(\left[b_{B}-b\right] f_{j}^{\infty}\right)(x)\right|^{2}\right)^{1 / 2} w(x) d x \\
& \leq \frac{w(B)}{\theta(w(B))} \cdot \frac{C}{\sigma} \sum_{l=1}^{\infty} \frac{1}{\left|2^{l+1} B\right|} \int_{2^{l+1} B}\left|b(z)-b_{B}\right| \cdot\left(\sum_{j}\left|f_{j}(z)\right|^{2}\right)^{1 / 2} d z
\end{aligned}
$$




$$
\begin{aligned}
& \leq \frac{w(B)}{\theta(w(B))} \cdot \frac{C}{\sigma} \sum_{l=1}^{\infty} \frac{1}{\left|2^{l+1} B\right|} \int_{2^{l+1} B}\left|b(z)-b_{2^{l+1} B}\right| \cdot\left(\sum_{j}\left|f_{j}(z)\right|^{2}\right)^{1 / 2} d z \\
& +\frac{w(B)}{\theta(w(B))} \cdot \frac{C}{\sigma} \sum_{l=1}^{\infty} \frac{1}{\left|2^{l+1} B\right|} \int_{2^{l+1} B}\left|b_{2^{l+1} B}-b_{B}\right| \cdot\left(\sum_{j}\left|f_{j}(z)\right|^{2}\right)^{1 / 2} d z \\
& :=K_{5}^{\prime}+K_{6}^{\prime} .
\end{aligned}
$$

For the term $K_{5}^{\prime}$, we first use the generalized Hölder's inequality with weight (2.6), (2.7) and (2.5) together with the $A_{1}$ condition to obtain

$$
\begin{aligned}
K_{5}^{\prime} & \leq \frac{C}{\sigma} \cdot \frac{w(B)}{\theta(w(B))} \sum_{l=1}^{\infty} \frac{1}{w\left(2^{l+1} B\right)} \int_{2^{l+1} B}\left|b(z)-b_{2^{l+1} B}\right| \cdot\left(\sum_{j}\left|f_{j}(z)\right|^{2}\right)^{1 / 2} w(z) d z \\
& \leq \frac{C}{\sigma} \cdot \frac{w(B)}{\theta(w(B))} \sum_{l=1}^{\infty}\left\|b-b_{2^{l+1} B}\right\|_{\exp L(w), 2^{l+1} B}\left\|\left(\sum_{j}\left|f_{j}\right|^{2}\right)^{1 / 2}\right\|_{L \log L(w), 2^{l+1} B} \\
& \leq \frac{C\|b\|_{*}}{\sigma} \cdot \frac{w(B)}{\theta(w(B))} \sum_{l=1}^{\infty} \inf _{\eta>0}\left\{\eta+\frac{\eta}{w\left(2^{l+1} B\right)} \int_{2^{l+1} B} \Phi\left(\frac{\|\vec{f}(z)\|_{\ell^{2}}}{\eta}\right) \cdot w(z) d z\right\} .
\end{aligned}
$$

Moreover, notice that the inequality $\Phi(a \cdot b) \leq \Phi(a) \cdot \Phi(b)$ holds for any $a, b>0$, when $\Phi(t)=t \cdot\left(1+\log ^{+} t\right)$. For $l=1,2, \ldots$, we may choose $\eta=\frac{\sigma \cdot \theta\left(w\left(2^{l+1} B\right)\right)}{w\left(2^{l+1} B\right)}$ and then use the estimate (3.7) to obtain

$$
\begin{aligned}
K_{5}^{\prime} & \leq \frac{C\|b\|_{*}}{\sigma} \cdot \frac{w(B)}{\theta(w(B))} \\
\times & \sum_{l=1}^{\infty}\left\{\sigma \cdot \frac{\theta\left(w\left(2^{l+1} B\right)\right)}{w\left(2^{l+1} B\right)}+\frac{\sigma}{w\left(2^{l+1} B\right)} \cdot \frac{\theta\left(w\left(2^{l+1} B\right)\right)}{w\left(2^{l+1} B\right)} \cdot \Phi\left(\frac{w\left(2^{l+1} B\right)}{\theta\left(w\left(2^{l+1} B\right)\right)}\right) \int_{2^{l+1} B} \Phi\left(\frac{\|\vec{f}(z)\|_{\ell^{2}}}{\sigma}\right) \cdot w(z) d z\right\} \\
& \leq C\|b\|_{*} \cdot\left[1+\sup _{B}\left\{\frac{\Phi\left(\frac{w(B)}{\theta(w(B))}\right)}{w(B)} \int_{B} \Phi\left(\frac{\|\vec{f}(z)\|_{\ell^{2}}}{\sigma}\right) \cdot w(z) d z\right\}\right] \\
& \times \sum_{l=1}^{\infty} \frac{\theta\left(w\left(2^{l+1} B\right)\right)}{\theta(w(B))} \cdot \frac{w(B)}{w\left(2^{l+1} B\right)} \\
& \leq C \cdot \sup _{B}\left\{\frac{\Phi\left(\frac{w(B)}{\theta(w(B))}\right)}{w(B)} \int_{B} \Phi\left(\frac{\|\vec{f}(z)\|_{\ell^{2}}}{\sigma}\right) \cdot w(z) d z\right\} .
\end{aligned}
$$

For the last term $K_{6}^{\prime}$ we proceed as follows. An application of the inequality (4.4) leads to that

$$
\begin{aligned}
K_{6}^{\prime} & \leq C \cdot \frac{w(B)}{\theta(w(B))} \sum_{l=1}^{\infty}(l+1)\|b\|_{*} \cdot \frac{1}{\left|2^{l+1} B\right|} \int_{2^{l+1} B} \frac{\|\vec{f}(z)\|_{\ell^{2}}}{\sigma} d z \\
& \leq C \cdot \frac{w(B)}{\theta(w(B))} \sum_{l=1}^{\infty}(l+1)\|b\|_{*} \cdot \frac{1}{w\left(2^{l+1} B\right)} \int_{2^{l+1} B} \frac{\|\vec{f}(z)\|_{\ell^{2}}}{\sigma} \cdot w(z) d z
\end{aligned}
$$




$$
\begin{aligned}
& \leq C \cdot \sup _{B}\left\{\frac{\Phi\left(\frac{w(B)}{\theta(w(B))}\right)}{w(B)} \int_{B} \Phi\left(\frac{\|\vec{f}(z)\|_{\ell^{2}}}{\sigma}\right) \cdot w(z) d z\right\} \\
& \times \sum_{l=1}^{\infty}(l+1) \cdot \frac{\theta\left(w\left(2^{l+1} B\right)\right)}{\theta(w(B))} \cdot \frac{w(B)}{w\left(2^{l+1} B\right)} .
\end{aligned}
$$

Notice that $w \in A_{1} \subset A_{\infty}$, by using the $\mathcal{D}_{\kappa}$ condition (2.9) of $\theta$ and the inequality (2.3) again together with the fact that $0 \leq \kappa<1$, we thus have

$$
\begin{aligned}
\sum_{l=1}^{\infty}(l+1) \cdot \frac{\theta\left(w\left(2^{l+1} B\right)\right)}{\theta(w(B))} \cdot \frac{w(B)}{w\left(2^{l+1} B\right)} & \leq C \sum_{l=1}^{\infty}(l+1) \cdot \frac{w(B)^{1-\kappa}}{w\left(2^{l+1} B\right)^{1-\kappa}} \\
& \leq C \sum_{l=1}^{\infty}(l+1) \cdot\left(\frac{|B|}{\left|2^{l+1} B\right|}\right)^{\delta^{*}(1-\kappa)} \\
& \leq C \sum_{l=1}^{\infty}(l+1) \cdot\left(\frac{1}{2^{(l+1) n}}\right)^{\delta^{*}(1-\kappa)} \\
& \leq C .
\end{aligned}
$$

Substituting the above inequality (6.2) into the term $K_{6}^{\prime}$, we finally obtain

$$
K_{6}^{\prime} \leq C \cdot \sup _{B}\left\{\frac{\Phi\left(\frac{w(B)}{\theta(w(B))}\right)}{w(B)} \int_{B} \Phi\left(\frac{\|\vec{f}(z)\|_{\ell^{2}}}{\sigma}\right) \cdot w(z) d z\right\} .
$$

Summing up all the above estimates, we therefore conclude the proof of the main theorem.

Further remark. Let $p=1,0 \leq \kappa<1, \theta$ satisfy the $\mathcal{D}_{\kappa}$ condition (2.9) and $w$ be a weight function on $\mathbb{R}^{n}$. We denote by $\mathcal{M}_{L}^{1, \theta} \log L(w)$ the generalized weighted Morrey space of $L \log L$ type, the space of all locally integrable functions $f$ defined on $\mathbb{R}^{n}$ with finite norm $\|f\|_{\mathcal{M}_{L \log L}^{1, \theta}(w)}$.

$$
\mathcal{M}_{L \log L}^{1, \theta}(w):=\left\{f \in L_{l o c}^{1}(w):\|f\|_{\mathcal{M}_{L \log L}^{1, \theta}(w)}<\infty\right\}
$$

where $\Phi(t)=t \cdot\left(1+\log ^{+} t\right)$ and

$$
\|f\|_{\mathcal{M}_{L \log L}^{1, \theta}(w)}:=\sup _{B}\left\{\frac{\Phi\left(\frac{w(B)}{\theta(w(B))}\right)}{w(B)} \int_{B}|f(x)| w(x) d x\right\},
$$

or

$$
\|f\|_{\mathcal{M}_{L \log L}^{1, \theta}(w)}:=\sup _{B}\left\{\frac{1+\log ^{+}\left(\frac{w(B)}{\theta(w(B))}\right)}{\theta(w(B))} \int_{B}|f(x)| w(x) d x\right\} .
$$


Obviously, we have $\mathcal{M}^{1, \theta}(w) \supseteq \mathcal{M}_{L \log L}^{1, \theta}(w)$ by the definition. Then the corresponding estimate in Theorem 2.5 reads

$\frac{1}{\theta(w(B))} \cdot w\left(\left\{x \in B:\left(\sum_{j}\left|\left[b, \mathcal{S}_{\alpha}\right]\left(f_{j}\right)(x)\right|^{2}\right)^{1 / 2}>\sigma\right\}\right) \leq C \cdot\left\|\Phi\left(\frac{\|\vec{f}\|_{\ell^{2}}}{\sigma}\right)\right\|_{\mathcal{M}_{L \log L}^{1, \theta}(w)}$.

Roughly speaking, we can say that the vector-valued commutator generated with $B M O$ function is bounded from $\mathcal{M}_{L \log L}^{1, \theta}(w)$ to $W \mathcal{M}^{1, \theta}(w)$ from the above definitions. In comparison with the conclusions of Theorems 2.4 and 2.5, it is natural to ask the question whether or not this vector-valued commutator has a more refined estimate:

$\frac{1}{\theta(w(B))} \cdot w\left(\left\{x \in B:\left(\sum_{j}\left|\left[b, \mathcal{S}_{\alpha}\right]\left(f_{j}\right)(x)\right|^{2}\right)^{1 / 2}>\sigma\right\}\right) \leq C \cdot\left\|\Phi\left(\frac{\|\vec{f}\|_{\ell^{2}}}{\sigma}\right)\right\|_{\mathcal{M}^{1, \theta}(w)}$.

By the technique used in this article, there is an "extra" $\log ^{+}(\cdot)$ term in the estimation of $K_{5}^{\prime}$. Whether this term can be removed is not known in general.

\section{References}

[1] J. Alvarez, R. J. Bagby, D. S. Kurtz and C. Pérez, Weighted estimates for commutators of linear operators, Studia Math, 104(1993), 195-209.

[2] Y. Ding, S. Z. Lu and K. Yabuta, On commutators of Marcinkiewicz integrals with rough kernel, J. Math. Anal. Appl, 275(2002), 60-68.

[3] J. Duoandikoetxea, Fourier Analysis, American Mathematical Society, Providence, Rhode Island, 2000.

[4] J. Garcia-Cuerva and J. L. Rubio de Francia, Weighted Norm Inequalities and Related Topics, North-Holland, Amsterdam, 1985.

[5] F. John and L. Nirenberg, On functions of bounded mean oscillation, Comm. Pure Appl. Math, 14(1961), 415-426.

[6] Y. Komori and S. Shirai, Weighted Morrey spaces and a singular integral operator, Math. Nachr, 282(2009), 219-231.

[7] T. Mizuhara, Boundedness of some classical operators on generalized Morrey spaces, Harmonic Analysis, ICM-90 Satellite Conference Proceedings, Springer-Verlag, Tokyo, (1991), 183-189.

[8] C. B. Morrey, On the solutions of quasi-linear elliptic partial differential equations, Trans. Amer. Math. Soc, 43(1938), 126-166.

[9] B. Muckenhoupt, Weighted norm inequalities for the Hardy maximal function, Trans. Amer. Math. Soc, 165(1972), 207-226. 
[10] C. Pérez, Endpoint estimates for commutators of singular integral operators, J. Funct. Anal, 128(1995), 163-185.

[11] C. Pérez and G. Pradolini, Sharp weighted endpoint estimates for commutators of singular integrals, Michigan Math. J., 49(2001), 23-37.

[12] C. Pérez and R. Trujillo-González, Sharp weighted estimates for multilinear commutators, J. London Math. Soc., 65(2002), 672-692.

[13] C. Pérez and R. Trujillo-González, Sharp weighted estimates for vectorvalued singular integral operators and commutators, Tohoku Math. J., 55(2003), 109-129.

[14] M. M. Rao and Z. D. Ren, Theory of Orlicz Spaces, Marcel Dekker, New York, 1991.

[15] E. M. Stein, Singular Integrals and Differentiability Properties of Functions, Princeton Univ. Press, Princeton, New Jersey, 1970.

[16] H. Wang, Intrinsic square functions on the weighted Morrey spaces, J. Math. Anal. Appl, 396(2012), 302-314.

[17] H. Wang, Boundedness of vector-valued intrinsic square functions in Morrey type spaces, J. Funct. Spaces., Article ID 923680, (2014), 8 pages.

[18] M. Wilson, The intrinsic square function, Rev. Mat. Iberoamericana, 23(2007), 771-791.

[19] M. Wilson, Weighted Littlewood-Paley Theory and Exponential-Square Integrability, Lecture Notes in Math, Vol 1924, Springer-Verlag, 2007.

[20] P. Zhang, Weighted endpoint estimates for commutators of Marcinkiewicz integrals, Acta Math. Sinica (Engl. Ser), 26(2010), 1709-1722. 